\title{
NEW YOUNG STAR CANDIDATES IN CG4 AND Sa101
}

\author{
L. M. Rebull ${ }^{1}$, C. H. Johnson ${ }^{2}$, V. Hoette ${ }^{3}$, J. S. Kim ${ }^{4}$, S. Laine ${ }^{1}$, M. Foster ${ }^{4}$, R. Laher ${ }^{1}$, \\ M. Legassie ${ }^{1,5}$, C. R. Mallory ${ }^{6}$, K. McCarron ${ }^{7}$, AND W. H. Sherry ${ }^{8}$ \\ ${ }^{1}$ Spitzer Science Center/Caltech, M/S 220-6, 1200 E. California Blvd., Pasadena, CA 91125, USA; luisa.rebull@jpl.nasa.gov \\ ${ }^{2}$ Breck School Science Department, 123 Ottawa Ave., N., Golden Valley, MN 55422, USA \\ ${ }^{3}$ The University of Chicago Department of Astronomy and Astrophysics, Yerkes Observatory Education and Outreach, \\ 373 W. Geneva St., Williams Bay, WI 53191, USA \\ ${ }^{4}$ Steward Observatory, University of Arizona, 933 N. Cherry Ave., Tucson, AZ 85721, USA \\ ${ }^{5}$ Raytheon Mission Operations \& Services, 299 N. Euclid Ave., Pasadena, CA 91101, USA \\ ${ }^{6}$ Department of Astronomy, Pierce College, 6201 Winnetka Ave., Woodland Hills, CA 91371, USA \\ ${ }^{7}$ Science Division, Oak Park and River Forest High School, 201 N. Scoville Ave., Oak Park, IL 60302, USA \\ ${ }^{8}$ NOAO/NSO, 950 N. Cherry Ave., Tucson, AZ 85719, USA \\ Received 2011 March 24; accepted 2011 April 29; published 2011 June 13
}

\begin{abstract}
The CG4 and Sa101 regions together cover a region of $\sim 0.5 \mathrm{deg}^{2}$ in the vicinity of a "cometary globule" that is part of the Gum Nebula. There are seven previously identified young stars in this region; we have searched for new young stars using mid- and far-infrared data $(3.6-70 \mu \mathrm{m})$ from the Spitzer Space Telescope, combined with ground-based optical data and near-infrared data from the Two Micron All Sky Survey. We find infrared excesses in all six of the previously identified young stars in our maps and identify 16 more candidate young stars based on apparent infrared excesses. Most (73\%) of the new young stars are Class II objects. There is a tighter grouping of young stars and young star candidates in the Sa101 region, in contrast to the CG4 region, where there are fewer young stars and young star candidates, and they are more dispersed. Few likely young objects are found in the "fingers" of the dust being disturbed by the ionization front from the heart of the Gum Nebula.
\end{abstract}

Key words: circumstellar matter - infrared: stars - stars: formation - stars: pre-main sequence

Online-only material: color figures

\section{INTRODUCTION}

Hawarden \& Brand (1976) identified "several elongated, comet-like objects" in the Gum Nebula. These objects have dense, dark, dusty heads and long, faint tails, that are generally pointing away from the center of the Vela OB2 association. More such "cometary globules" (CGs) were subsequently identified in the Gum Nebula (e.g., Sandqvist 1976; Reipurth 1983), but similar structures had also been identified elsewhere (e.g., Orion, Rosette Nebula, etc.) in the context of bok globules (e.g., Bok \& Reilly 1947) and "elephant trunks" (e.g., Osterbrock 1957). These objects are all thought to be related in the following sense-certain regions of the molecular cloud are dense enough to persist when the stellar winds and ionizing radiation from $\mathrm{OB}$ stars powering an $\mathrm{H}$ II region move over them, initially forming elephant trunks and then eventually CGs. These structures often also have bright rims, thought to originate from the OB stars' winds and radiation, and are often actively forming stars (e.g., Reipurth 1983), most likely triggered by the interaction with winds and radiation from the OB stars (e.g., Haikala et al. 2010).

Cometary globule 4 (CG4) in the Gum Nebula has a striking appearance (see Figures 1-5), where the combination of the original dust distribution plus ablation from the $\mathrm{OB}$ winds and radiation has resulted in a relatively complicated structure. A serendipitous placement of background galaxy ESO 257-G 019 just 0.15 to the east of the heart of CG4 adds to the drama of the image (see Figures 1-5). About half of a degree to the west of the heart of CG4 is another cloud named Sa101. This region was initially recognized by Sandqvist (1977) as an opacity class 5 (on a scale of 6, e.g., fairly dark) dark cloud. This cloud appears to have been shadowed, at least partially, by CG4 from the ionization front (see the discussion in Pettersson 2008).
Reipurth \& Pettersson (1993) studied the region of CG4+Sa101, finding several $\mathrm{H} \alpha$ emission stars; see Table 1. They point out that CG-H $\alpha 1$ and 7 are not associated with dusty material and, as such, may have been associated with clumps that have already evaporated, as opposed to the stars still projected onto molecular cloud material. They argue on the basis of $\mathrm{H} \alpha$ equivalent widths that these cannot be foreground or background dMe stars, but are instead likely young stars, members of the association. They then use this to argue that this cloud is most likely a part of the Gum Nebula Complex (as opposed to a foreground or background object). We therefore assume that the stars associated with CG4+Sa101 are also associated with the Gum Nebula.

Distances to CG4+Sa101 and even the Gum Nebula are uncertain, with values between 300 and $500 \mathrm{pc}$ appearing in the literature (e.g., Franco 1990). The generally accepted source of strong ultraviolet radiation is $\gamma^{2}$ Velorum, which is taken to be 360-490 pc away (Pozzo et al. 2000). However, the Gum Nebula is elongated along our line of sight, so the distance to different parts of the nebula could be significantly different than the distance to $\gamma^{2}$ Velorum. Vela OB2 is $\sim 425 \mathrm{pc}$ (Pozzo et al. 2000). In the context of this paper, we test the extrema of the distance estimates of 300 and $500 \mathrm{pc}$, though we note that our results are not strongly dependent on distance.

Since the CG4+Sa101 region contains some previously identified young stars, it is likely that there are more young stars, perhaps lower mass or more embedded than those discovered previously. Kim et al. (2003), using a preliminary reduction of some of the optical data used here, discussed some additional candidate young stars in this region. Since it is now commonly believed that every low-mass star goes through a period of having a circumstellar disk, young stars can be identified via an 


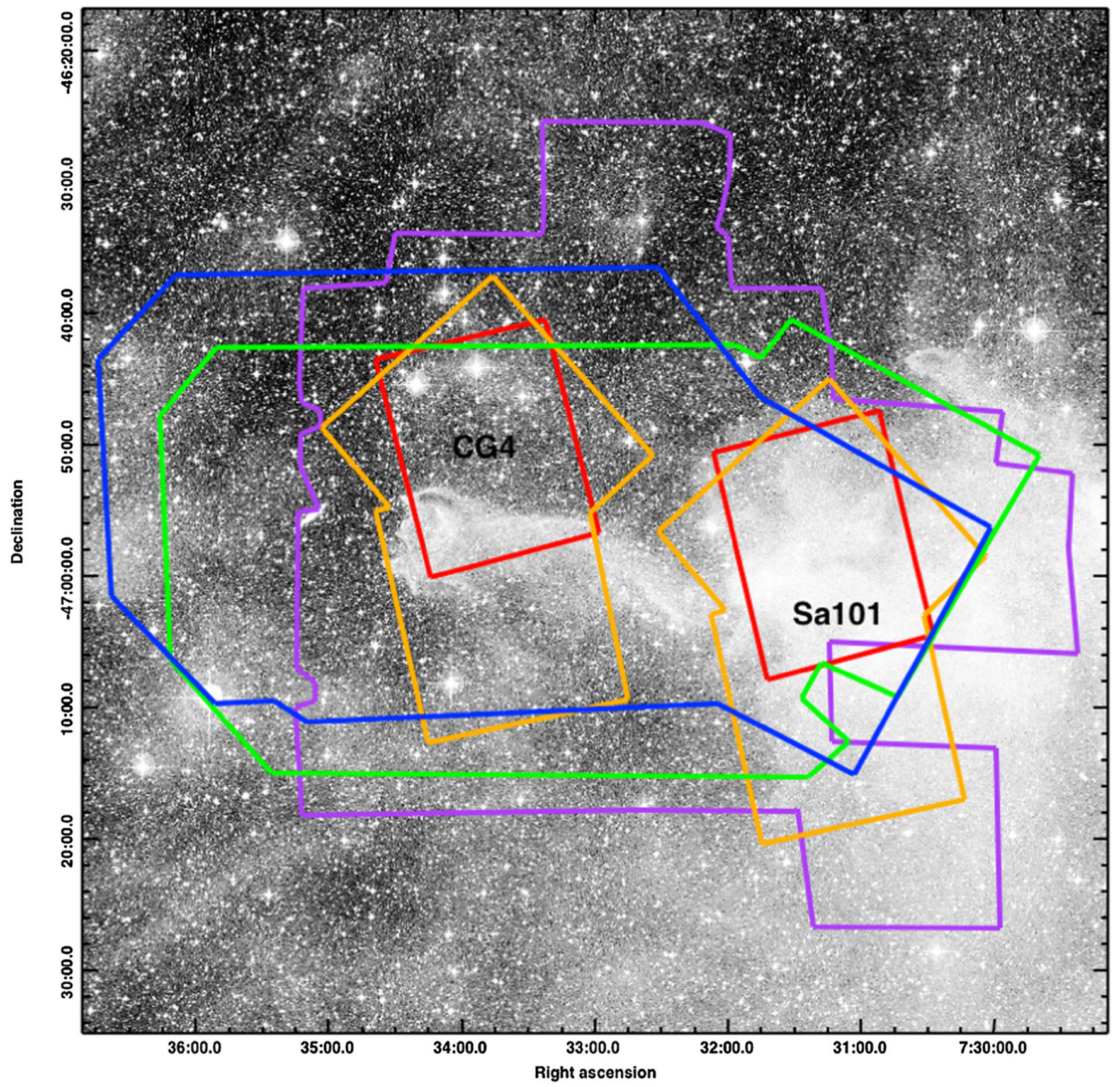

Figure 1. Approximate location of optical, IRAC, MIPS coverage, superimposed on a Palomar Observatory Sky Survey (POSS) image. Purple is optical, blue is IRAC-1 and -3, green is IRAC-2 and -4, orange is MIPS-1, and red is MIPS-2. The approximate locations of CG4 and Sa101 are also indicated. The galaxy ESO 257-G 019 is located on the left, partially obscured by the edge of the optical coverage.

Table 1

Previously Identified Young Stars in the CG4+Sa101 Region ${ }^{\mathrm{a}}$

\begin{tabular}{|c|c|c|c|c|c|c|c|c|c|c|}
\hline Name & Region & R.A. (J2000) & Decl. (J2000) & $U(\mathrm{mag})$ & $B(\mathrm{mag})$ & $V(\mathrm{mag})$ & $J(\mathrm{mag})$ & $H$ (mag) & $K(\mathrm{mag})$ & Spec. Type \\
\hline$\overline{\mathrm{CG}-\mathrm{H} \alpha 1^{\mathrm{b}}}$ & SA 101 & 073037.6 & -472506 & $\cdots$ & $\ldots$ & $>17$ & $\ldots$ & $\ldots$ & $\ldots$ & M3-4 \\
\hline $\mathrm{CG}-\mathrm{H} \alpha 2$ & SA 101 & 073057.5 & -465611 & $\ldots$ & $\ldots$ & $>17$ & $\ldots$ & $\ldots$ & $\ldots$ & M2: \\
\hline CG-H $\alpha 3$ & SA 101 & 073110.8 & -470032 & 17.50 & 16.59 & 14.99 & 11.51 & 10.35 & 9.62 & K7 \\
\hline CG-H $\alpha 4$ & SA 101 & 073121.8 & -465745 & 16.91 & 15.99 & 14.59 & 11.21 & 10.38 & 9.91 & K7-M0 \\
\hline $\mathrm{CG}-\mathrm{H} \alpha 5$ & SA 101 & 073136.6 & -470013 & 16.74 & 16.51 & 15.25 & 11.73 & 10.64 & 9.96 & $\mathrm{~K} 2-5$ \\
\hline $\mathrm{CG}-\mathrm{H} \alpha 6$ & SA 101 & 073137.4 & -470021 & 16.53 & 15.63 & 14.21 & 10.42 & 9.52 & 9.06 & K7 \\
\hline $\mathrm{CG}-\mathrm{H} \alpha 7$ & CG 4 & 073326.8 & -464842 & 16.00 & 15.16 & 13.97 & $\ldots$ & $\ldots$ & $\ldots$ & K5 \\
\hline
\end{tabular}

Notes.

${ }^{a}$ Information tabulated here comes from Reipurth \& Pettersson (1993), with positions updated to be J2000 and tied to the Spitzer and 2MASS coordinate system. We assumed the errors on the photometry to be $\sim 20 \%$ when plotting them in the spectral energy distributions (SEDs) in Figures 14-16.

${ }^{\mathrm{b}}$ Off the edge of the Spitzer maps discussed here. 


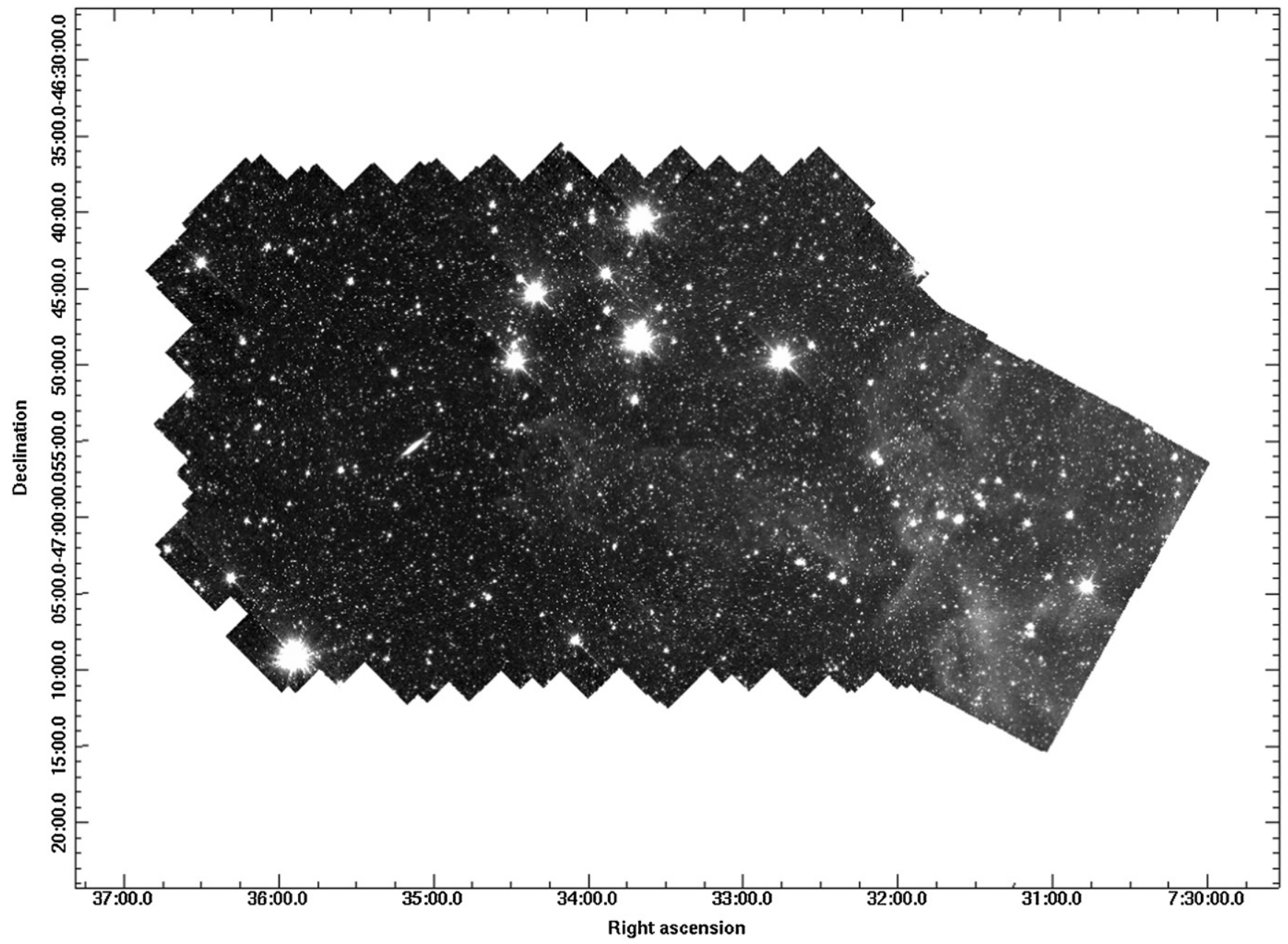

Figure 2. IRAC $3.6 \mu \mathrm{m}$ (channel 1) mosaic.

infrared (IR) excess, assumed to be due to a circumstellar disk. A survey in the IR can be used to identify objects having an IR excess and thus distinguish candidate young stars from most foreground or background objects, at least those foreground or background stars without circumstellar disks. The IR also more easily penetrates the dusty environs of star-forming regions, particularly globules such as these CGs in the Gum Nebula.

The Spitzer Space Telescope (Werner et al. 2004) observed the CG4+Sa101 region with IRAC (Fazio et al. 2004) at 3.6, 4.5, 5.8, and $8 \mu \mathrm{m}$, and with MIPS (Rieke et al. 2004) at 24 and $70 \mu \mathrm{m}$. We used these data to search this region for additional young stellar object (YSO) candidates. We combined these Spitzer data with data from the near-infrared Two Micron All Sky Survey (2MASS; Skrutskie et al. 2006) and from ground-based optical photometric data that we have obtained, and used the multiwavelength catalog to evaluate and rank our list of Spitzerselected YSO candidates.

The observations and data reduction are described in Section 2. We select YSO candidates using Spitzer colors in Section 3 and discuss their overall properties in Section 4 . We include a few words on the serendipitously observed galaxy in Section 5. Finally, we summarize our main points in Section 6.

\section{OBSERVATIONS, DATA REDUCTION, AND ANCILLARY DATA}

In this section, we discuss the IRAC and MIPS data acquisition and reduction. We briefly summarize the optical $\left(B V R_{c} I_{c}\right)$ data reduction, which will be covered in more detail in $\mathbf{J}$. S. Kim et al. (2011, in preparation). We also discuss merging the photometric data across bands, and with the 2MASS near-IR catalog $\left(J H K_{s}\right)$. The regions of the sky covered by IRAC, MIPS, and the optical observations are indicated in Figure 1.

We note for completeness that the four channels of IRAC are $3.6,4.5,5.8$, and $8 \mu \mathrm{m}$ and that the three channels of MIPS are 24,70 , and $160 \mu \mathrm{m}$. These bands can be referred to equivalently by their channel number or wavelength; the bracket notation, e.g., [24], denotes the measurement in magnitudes rather than flux density units (e.g., Jy). Further discussion of the bandpasses can be found in, e.g., the Instrument Handbooks, available from the Spitzer Science Center (SSC) or the Infrared Science Archive (IRSA) Spitzer Heritage Archive (SHA) Web sites.

\subsection{IRAC Data}

We used the IRAC data for CG4 from program 462, AORKEY $^{9}$ 24250880; for Sa101, we used the IRAC data from program 20714, AORKEY 16805888. The IRAC data from program 462 (for CG4) were taken on 2007 December 29 with $12 \mathrm{~s}$ high-dynamic-range (HDR) frames, so there are two exposures at each pointing, 0.6 and $12 \mathrm{~s}$, with three dithers per position, for a total integration time of $36 \mathrm{~s}$ (on average). The IRAC data from

\footnotetext{
9 An AOR is an Astronomical Observation Request, the fundamental unit of Spitzer observing. An AORKEY is the unique eight-digit identifier for the AOR, which can be used to retrieve these data from the Spitzer Archive.
} 


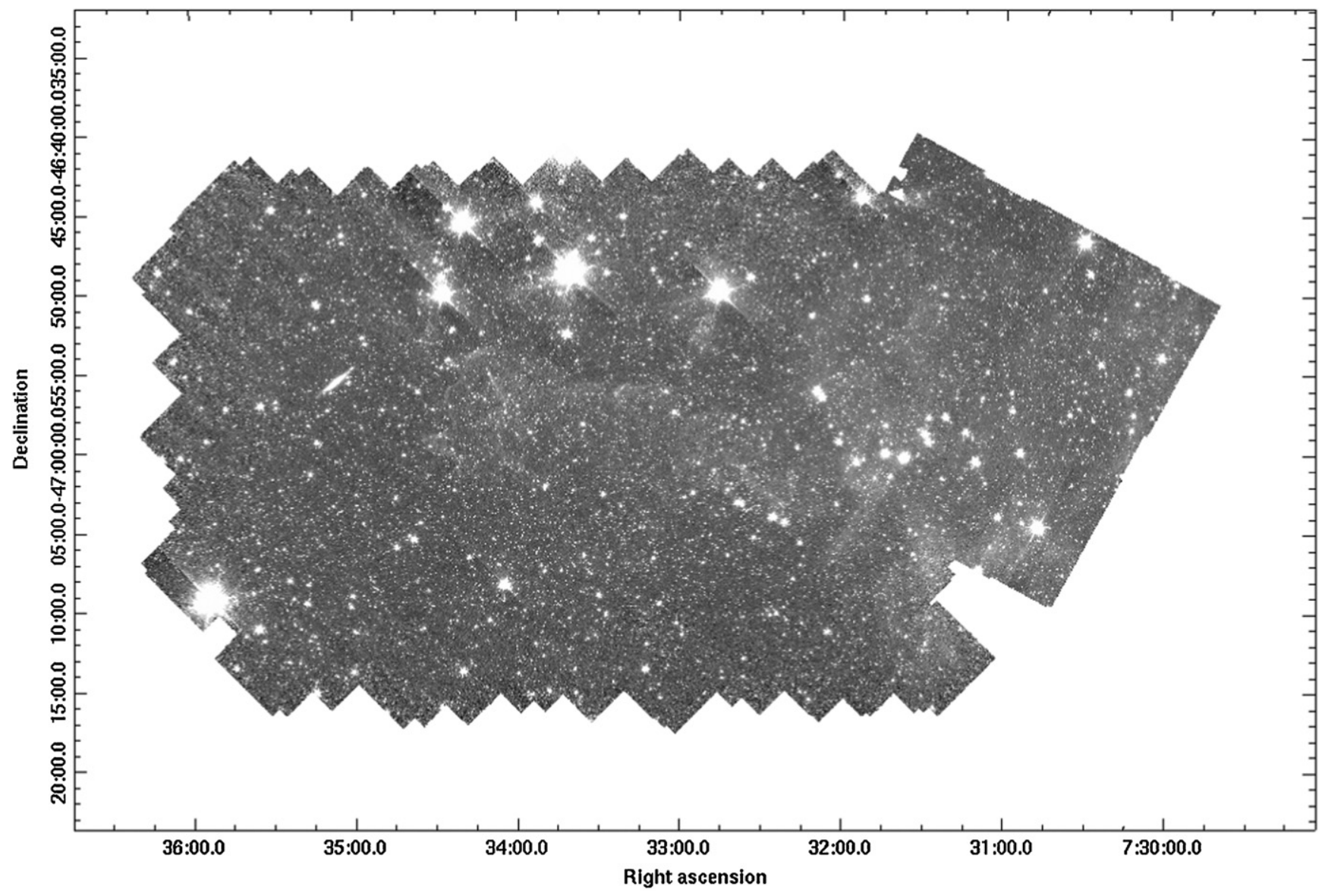

Figure 3. IRAC $4.5 \mu \mathrm{m}$ mosaic (channel 2).

program 20714 (for Sa101) were taken on 2006 March 27 with 30 s HDR frames; there are also two exposures per pointing, but deeper, 1.2 and $30 \mathrm{~s}$. For this observation, there are two dithers per position, for a total integration time of $60 \mathrm{~s}$ (on average). Because of the different integration times, we reduced the Sa101 and CG4 observations independently even though they overlap on the sky (in Figures 2-5, the jagged-edged observation is from the observation in program 462, and the smooth-edged observation on the right is from the observation in program 20714).

We note that there are additional IRAC data in this region that we did not use. IRAC data from program 202 were of a very small region centered on the head of the globule. We did not include these data in an effort to make our survey as uniform as possible over the entire surveyed region. IRAC data from program 20714 for CG4 were taken in non-HDR mode, with 30 s exposures; as a result of some very bright stars in the field of view, the instrumental effects rendered these data very difficult to work with. Our science goals near CG4 can be met with the total integration time from program 462 alone. In addition, the data from program 462 cover a larger area $\left(\sim 0.8 \times \sim 1^{\circ}\right)$ than from program $20714(\sim 0.4 \times \sim 0.5)$. For these reasons, we did not incorporate the CG4 IRAC data from program 20714 in this analysis.

We started with the corrected basic calibrated data (CBCD) processed using SSC pipeline version 18.7. Because of the very bright stars in the field of view, and because the data from program 462 were taken with cluster targets, we could not use the pipeline-processed mosaics. Moreover, the artifact correction, which is normally done for individual cluster targets separately in the SSC pipeline processing, is much improved when using the CBCD files from program 462 all at once. We reprocessed the IRAC data from both program 462 (for CG4) and program 20714 (for Sa101), using MOPEX (Makovoz \& Marleau 2005) to calculate overlap corrections and create mosaics with very much reduced instrumental artifacts compared to the pipeline mosaics. The pixel size for our mosaics was the same as the pipeline mosaics, 0.6 arcsec, half of the native pixel scale. We created separate mosaics for the long and the short exposures at each channel for photometric analysis. For display purposes, we further used MOPEX to combine the two longframe observations into one large mosaic per channel, as seen in Figures 2-5. The component mosaics were properly weighted in terms of signal-to-noise and exposure time. The total area covered by at least one IRAC channel (as seen in Figures 2-5) is $\sim 0.5 \mathrm{deg}^{2}$.

To obtain photometry of sources in this region, we used the APEX-1frame module from MOPEX to perform source detection on the resultant long and short mosaics for each observation separately. We took those source lists and used the aper.pro routine in IDL to perform aperture photometry on the mosaics with an aperture of 3 native pixels ( 6 resampled pixels), and an annulus of 3-7 native pixels (6-14 resampled pixels). The corresponding aperture corrections are, for the four IRAC channels, 1.124, 1.127, 1.143, and 1.234, respectively, as listed in the IRAC Instrument Handbook. As a check on the photometry, the educators and students associated with this project (see the acknowledgments) used the Aperture Photometry Tool (Laher et al. 2011a, 2011b) to confirm by hand the measurements for all the targets of interest. To convert the flux densities to magnitudes, we used the zero points as 


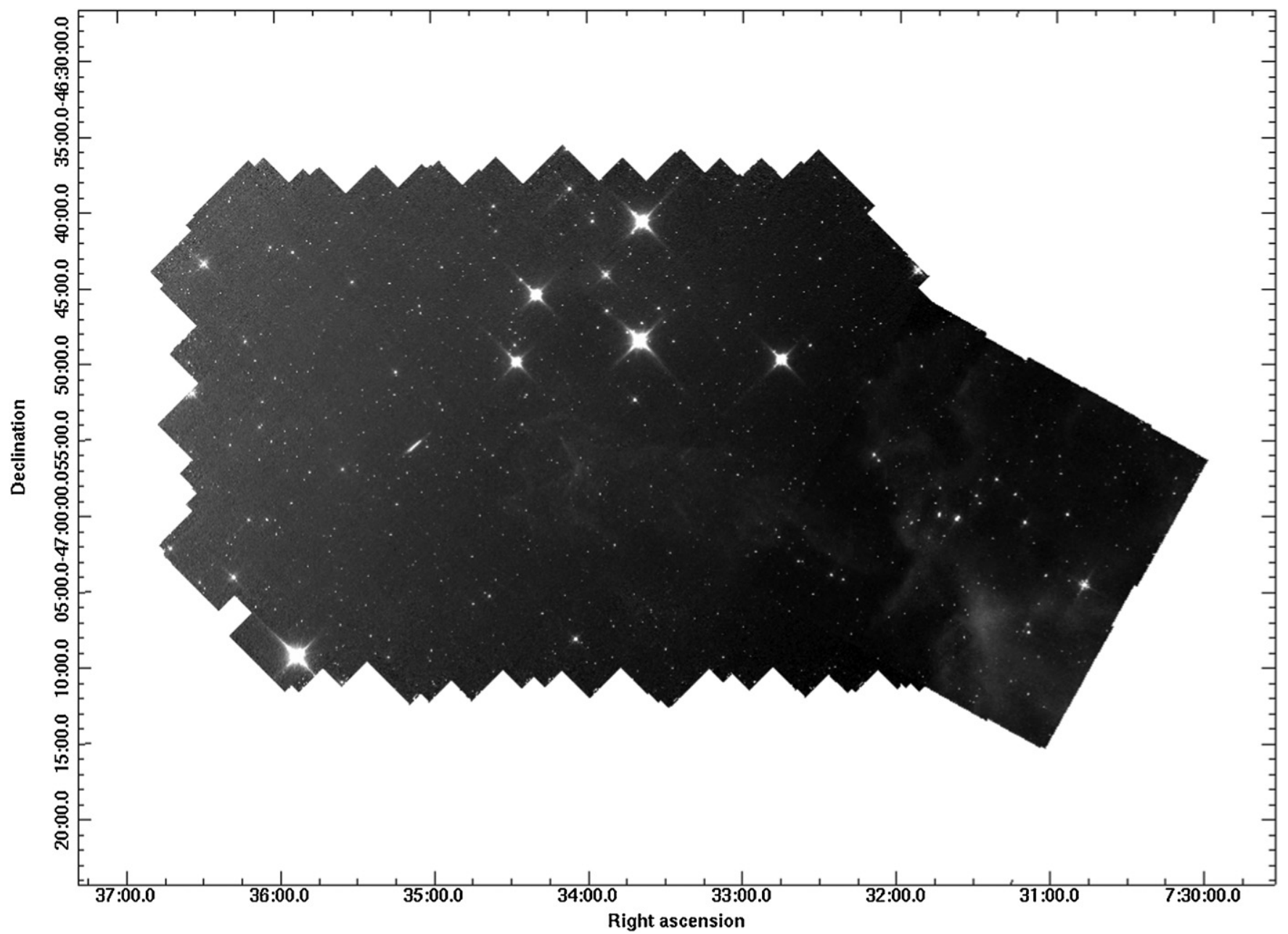

Figure 4. IRAC $5.8 \mu \mathrm{m}$ mosaic (channel 3).

provided in the IRAC Instrument Handbook: 280.9, 179.7, 115.0, and 64.13 Jy, respectively, for the four channels. (No array-dependent color corrections nor regular color corrections were applied.) We took the errors as produced by IDL to be the best possible internal error estimates; to compare to flux densities from other sources, we took a flat error estimate of 5\% added in quadrature.

To obtain one source list per channel per observation, we then merged the short and the long exposures for each channel separately, and for each observation independently, because of the different exposure times as noted above. The crossover points between taking fluxes from the short and long exposures were taken from empirical studies of prior star-forming regions and were magnitudes of 9.5, 9.0, 8.0, and 7.0 for the four IRAC channels, respectively. We performed this merging via a strict by-position search, looking for the closest match within $1^{\prime \prime}$. This maximum radius for matching was determined via experience with other star-forming regions (e.g., Rebull et al. 2010). The limiting magnitudes of these final source lists are the same for both observations and are [3.6] $17 \mathrm{mag}$, [4.5] $17 \mathrm{mag}$, [5.8] $15.5 \mathrm{mag}$, and [8] 14.5 mag.

\subsection{MIPS Data}

There are two MIPS AORs in the CG4 region and two MIPS AORs in the Sa101 region, all four of which were obtained as part of program 20714 (AORKEYs 16805632, 16807936,
16806144, and 16808192) on 2006 May 8 or June 12; see Figures 1, 6, and 7. The AORs were obtained in MIPS photometry mode, nominally centered on 7:31:18.7, $-46: 57: 45$ for Sa101 and 7:33:48 -46:49:59.9 for CG4. One of each pair of AORs is explicitly a MIPS-24 photometry observation, and the other is a MIPS-70 photometry observation. During the $70 \mu \mathrm{m}$ observation, the $24 \mu \mathrm{m}$ array is still turned on and is still collecting valid data. We combined the prime $24 \mu \mathrm{m}$ data from the MIPS-24 photometry-mode observations with the serendipitous $24 \mu \mathrm{m}$ data from the MIPS-70 photometry-mode observations to obtain larger maps at $24 \mu \mathrm{m}$. The original explicitly $24 \mu \mathrm{m}$ photometry observations are small photometry-mode maps, with $3 \mathrm{~s}$ integration per pointing, but a net integration time of $\sim 210 \mathrm{~s}$ over most of the resultant mosaic. The serendipitously obtained data averaged $\sim 350$ s net integration time; where the deliberate and serendipitous data overlapped, integration times can be $\sim 450 \mathrm{~s}$. The $70 \mu \mathrm{m}$ photometry observations were also small photometry-mode maps, with $10 \mathrm{~s}$ integration per pointing; the net integration time over most of the mosaics was $\sim 400 \mathrm{~s}$.

The data for $24 \mu \mathrm{m}$, like those for IRAC, were affected by the bright objects and required additional processing beyond what the online pipelines could provide. We started with S18.13 enhanced BCDs from the pipeline. We implemented a self-flat for each AOR separately, as described in the MIPS Instrument Handbook, available from the SSC or IRSA SHA Web sites. For each pair of overlapping $24 \mu \mathrm{m}$ maps, we then ran an overlap correction using the overlap script that comes with MOPEX 


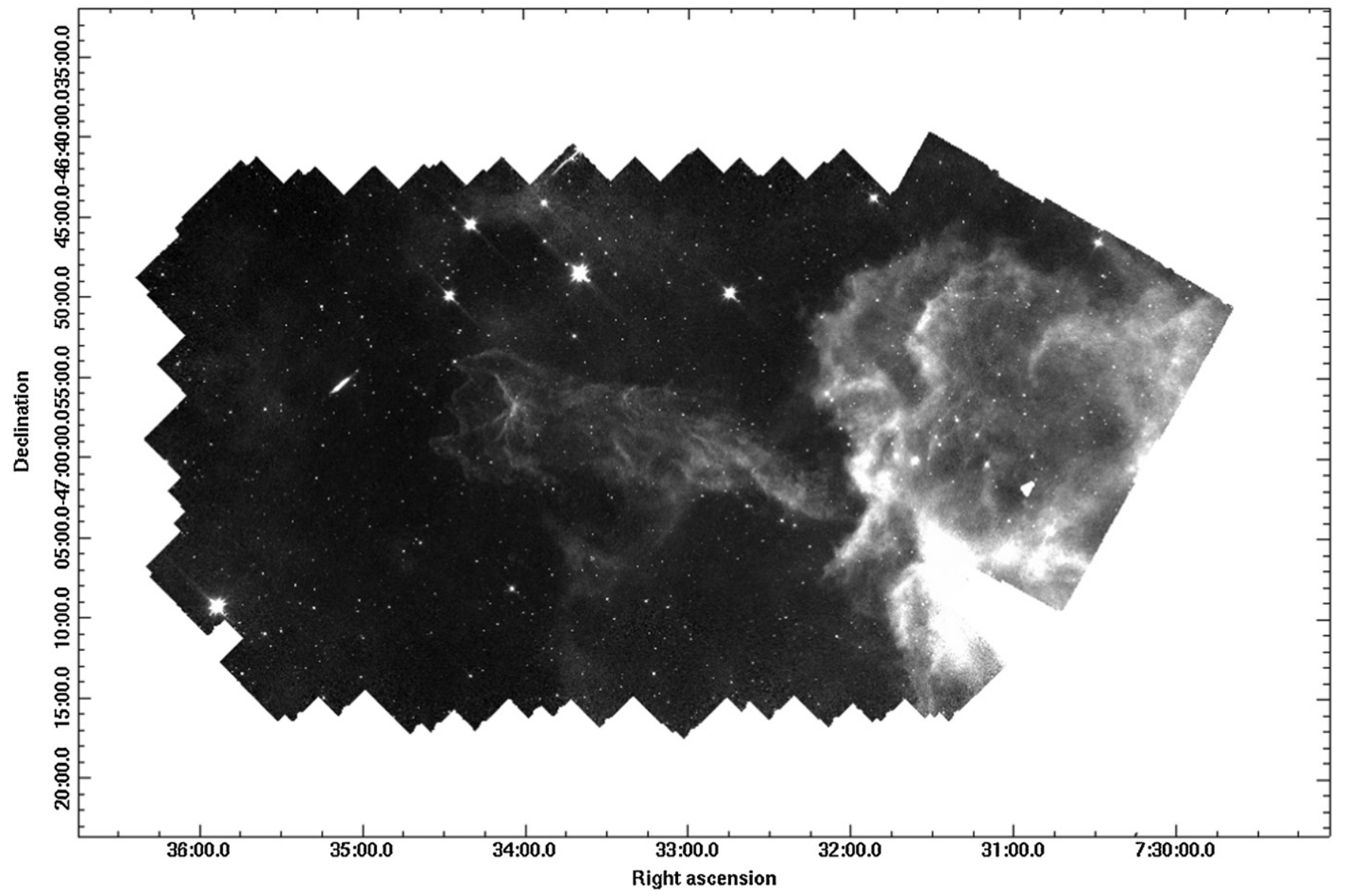

Figure 5. IRAC $8 \mu \mathrm{m}$ mosaic (channel 4).

and then created one mosaic for CG4 and one for Sa101, again using MOPEX. Our mosaics had the same pixel size as the online mosaics, 2". 45. In order to combine the images into one mosaic for display in Figure 6, the different overall background levels between the two observations (having an origin in the different zodiacal light levels at the times of the two observations) were problematic. The brighter of the two was artificially lowered via median subtraction to bring its dynamic range into a similar regime as the fainter; this renders photometry on this net mosaic invalid, but the morphology seen in Figure 6 is still valid. The total area covered by the net $24 \mu \mathrm{m}$ map is only $\sim 0.3 \mathrm{deg}^{2}$, smaller than that of the IRAC map.

To obtain photometry at $24 \mu \mathrm{m}$, we ran APEX-1frame on each of the mosaics (one per observation) and performed point-response-function (PRF) fitting photometry using the SSC-provided PRF. Tests using the apex_qa module portion of MOPEX suggest that our photometry is well within expected errors. For three problematic sources, we used aperture photometry instead of the PRF-fitted photometry, as they provided a better fit in apex_qa. We used the signal-to-noise ratio $(\mathrm{S} / \mathrm{N})$ value returned by APEX-1frame as the best estimate of the internal (statistical) errors, adding a $4 \%$ flux density error in quadrature as a best estimate of the absolute uncertainty. The limiting magnitude of these observations is [24] $10.5 \mathrm{mag}$. Note that we optimized our data reduction to obtain measurements of the brighter sources and sources superimposed on the nebulosity; many sources fainter than this are apparent in the image but not included in our catalog, simply because our scientific goals are aimed at the brighter objects. For one source of interest below (073243.5-464941, which was considered and then rejected as a YSO candidate; see Section 3), an upper limit was obtained at the given position by laying down an aperture as if a source were there, and taking three times that value for the $3 \sigma$ limit. To convert the flux densities to magnitudes, we used the zero point as found in the MIPS Instrument Handbook, $7.14 \mathrm{Jy}$.

At $70 \mu \mathrm{m}$, there are viable observations from AORKEYs 16807936 and 16808192 . We downloaded data processed with pipeline version S18.12. The online pipeline does a very good job of producing mosaics; see Figure 7, where there are a handful of point sources and extended emission visible. The online pipeline produces both filtered and unfiltered mosaics; the filtering preserves the flux densities of the point sources and improves their $\mathrm{S} / \mathrm{N}$, especially for faint sources, but destroys the flux density information for the extended emission. The unfiltered mosaics are shown in Figure 7, but we performed photometry on the filtered mosaics. The pipeline mosaics have resampled $4^{\prime \prime}$ pixels (as opposed to $5^{\prime \prime} .3$ native pixels), and the two observations together cover about $0.1 \mathrm{deg}^{2}$. We used APEX1 frame to do PRF fitting on the pipeline filtered mosaics for the point sources, using the SSC-provided PRF. For one problematic source, aperture photometry provided a better flux density estimate. There are only 11 objects with $70 \mu \mathrm{m}$ detections, and there is a large variation in background levels, so quoting a limiting magnitude is difficult, but is approximately 3 mag. We assumed a conservative, flat $20 \%$ flux density error. The zero point we used again came from the MIPS Instrument Handbook, $0.775 \mathrm{Jy}$. 


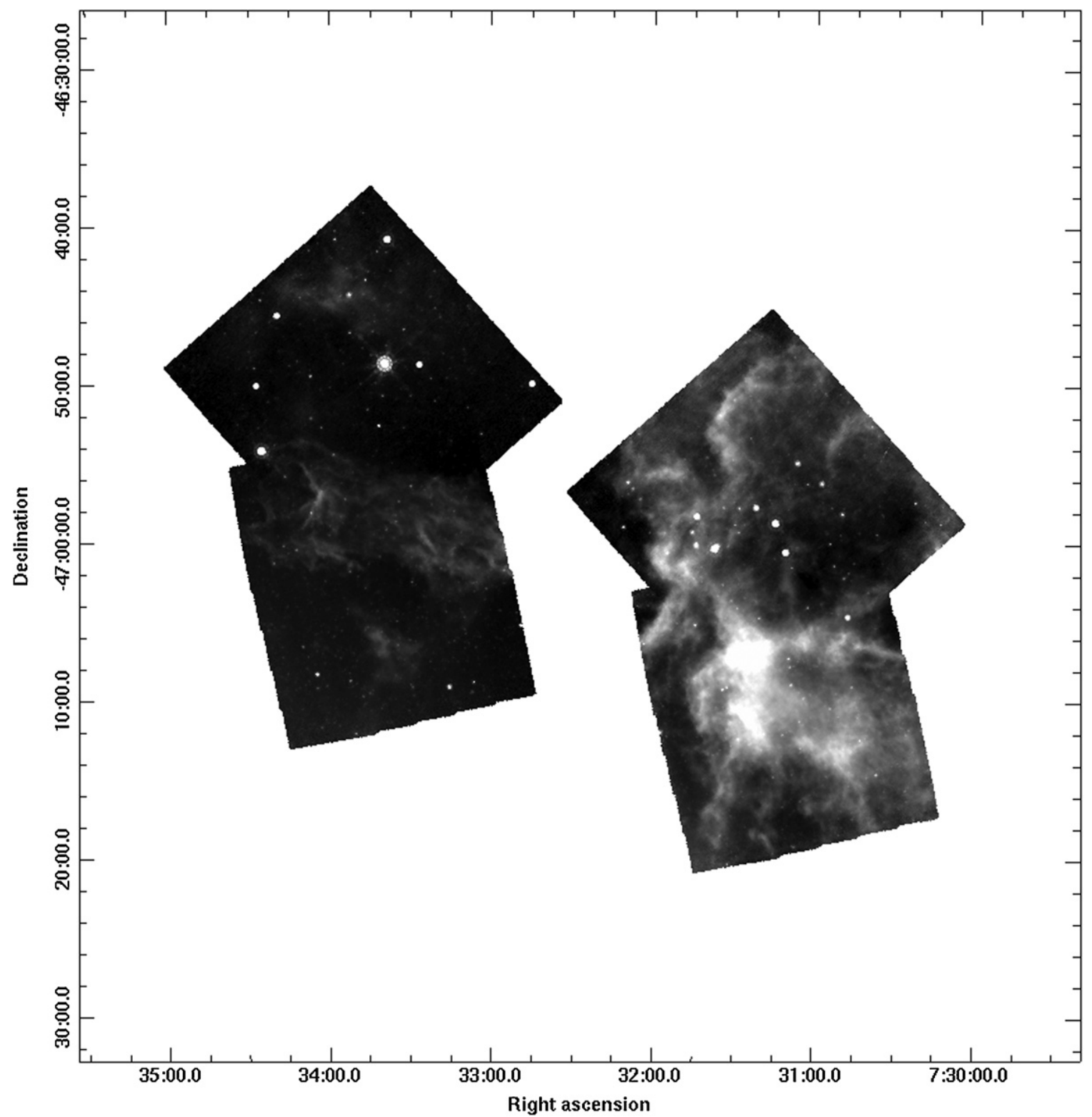

Figure 6. MIPS $24 \mu \mathrm{m}$ mosaic. The $24 \mu \mathrm{m}$ coverage consists of two pointed photometry-mode small maps, plus the $24 \mu \mathrm{m}$ data serendipitously obtained during $70 \mu \mathrm{m}$ photometry observations. Extended emission and point sources are both apparent.

Where there was $70 \mu \mathrm{m}$ coverage for the sources of interest, we placed an aperture at the expected location of the source and performed photometry as if there were a source there, taking three times that value as the $3 \sigma$ limit that appears in Table 2 below.

\subsection{Optical Data}

The optical data will be discussed further in J. S. Kim et al. (2011, in preparation), but we summarize the important aspects of the data reduction here.

The $B V R_{c} I_{c}$ photometry of the CG4+Sa101 region was obtained during 2001 March 6, 7, 9, 10, and 11 using the $2 \mathrm{~K} \times 2 \mathrm{~K} \mathrm{CCD}$ at the $0.9 \mathrm{~m}$ telescope at the Cerro Tololo Inter-American Observatory (CTIO). The images have a pixel scale of 0'.4 in a 13'.6 field of view.

Bias and twilight sky flat fields were taken at the beginning and at the end of each night. Long and short (300 s and $30 \mathrm{~s}$ ) exposures were taken for object fields. During every photometric night we observed Landolt (1992) standard stars of two or three fields several times per night for photometric calibration.

We performed aperture photometry using multiple aperture sizes, and the photometry with highest $\mathrm{S} / \mathrm{N}$ was chosen as final photometry for each star. For the standard stars, we used an aperture radius of 17 pixels. We used $\mathrm{IRAF}^{10} / \mathrm{PHOTCAL}$ routines to solve for the zero point, extinction, and color terms of the standard star solution.

For the target stars in CG4 and Sa101, we used a custom IDL photometry pipeline (written by W. H. Sherry), which was developed for the CTIO $0.9 \mathrm{~m}$ telescope. For each target, aperture photometry was performed using multiple size apertures starting from aperture size of 2 pixels to 17 pixels. The highest $\mathrm{S} / \mathrm{N}$ photometry was chosen as the final photometry.

10 IRAF is distributed by the National Optical Astronomy Observatory, which is operated by the Association of Universities for Research in Astronomy (AURA), Inc., under cooperative agreement with the National Science Foundation. 


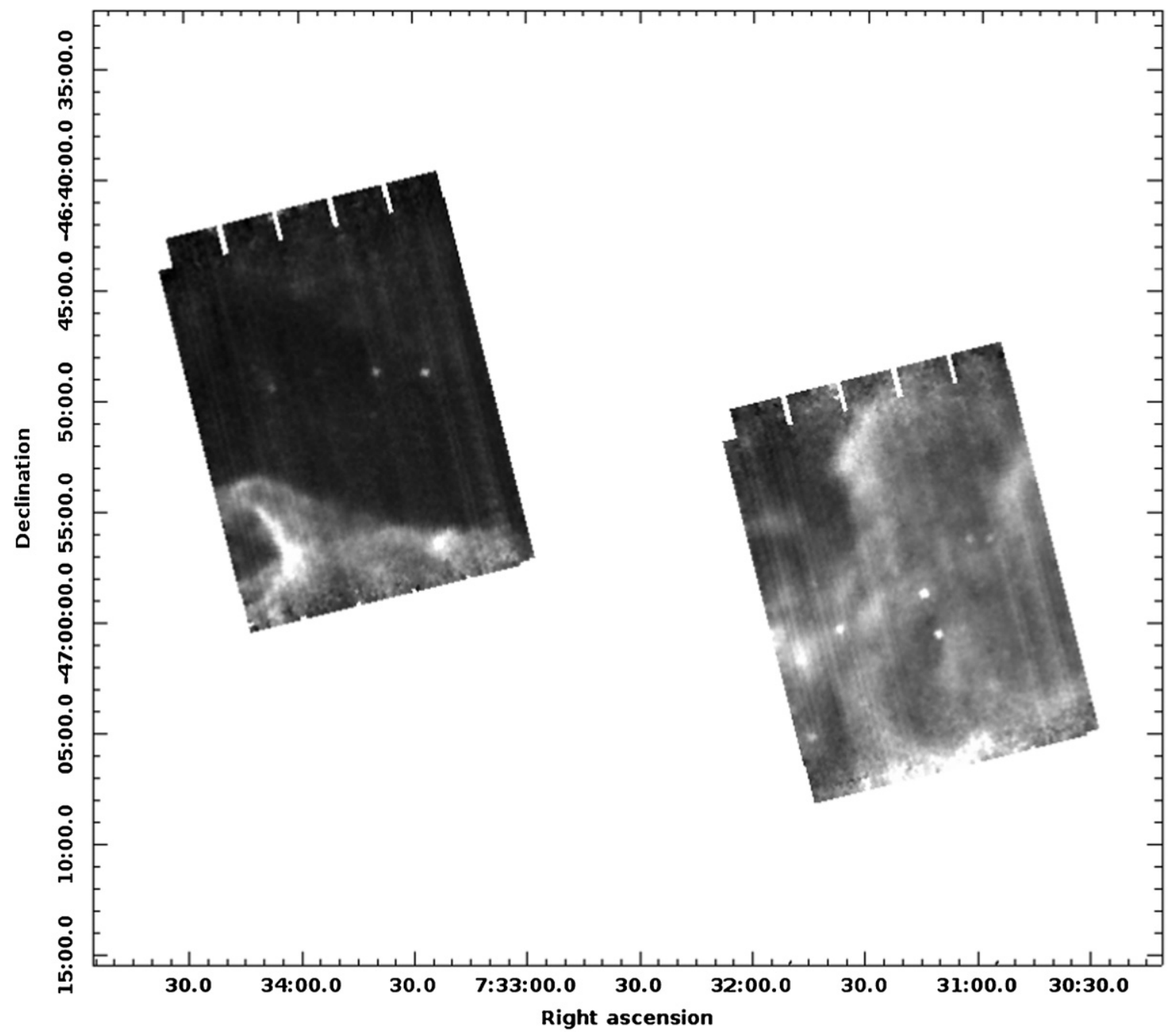

Figure 7. MIPS $70 \mu \mathrm{m}$ mosaic. The $70 \mu \mathrm{m}$ coverage is two pointed photometry-mode small maps. Extended emission and point sources are both apparent.

We used an aperture correction to place our photometry on the same photometry system as our standard stars. The point-spread function (PSF) of the CTIO $0.9 \mathrm{~m}$ telescope varies noticeably as a function of location on the CCD. This is insignificant for large apertures, but for aperture sizes of 2-3 pixels, the difference can be a few percent. We accounted for the spatial dependence of the aperture correction in each image by fitting the aperture corrections for stars with photometric errors better than $0.02 \mathrm{mag}$ with a quadratic function. The uncertainty of the aperture correction is about $0.01 \mathrm{mag}$. For each aperture, the uncertainty on the instrumental magnitude is calculated including the uncertainty on the aperture correction for each aperture.

We used the imwcs program written by D. Mink ${ }^{11}$ (Mink 1997) to determine the astrometric solution of each image. It fits the pixel coordinates of our targets to the known positions of USNO A2.0 stars located in each field. We then measured positions to an accuracy of $\sim 0^{\prime \prime} 3$ relative to the USNO A2.0 reference frame.

For each pointing, we used a $1^{\prime \prime}$ matching radius to match sources within the optical $B V R_{c} I_{C}$ filters. The coordinates are averaged over different filters, and the typical average uncertainty is $0.2-0.3$. To find duplicates between adjacent

\footnotetext{
11 Documentation and source code are available at http://tdc-www.harvard.edu/software/wcstools/.
}

pointings, we again looked for positional matches within $1^{\prime \prime}$ and then took a weighted mean of the available photometry.

Basically the entire IRAC map was covered west of 113.8 R.A. (07:35:12); see Figure 1. We have found no YSO candidates east of this. The completeness limits over the field are as follows: $B \sim 19 \mathrm{mag}, V \sim 18 \mathrm{mag}, R_{c} \sim 17.5 \mathrm{mag}$, and $I_{c} \sim 17$ mag. However, we note that there are fewer sources per projected area detected (e.g., effectively shallower limits) in the regions where there is molecular cloud material. The zero points we used for conversion of the magnitudes to flux densities (for inclusion in the spectral energy distributions (SEDs) in Figures 14-16) were, respectively, 4000.87, 3597.28, 2746.63, and $2432.84 \mathrm{Jy}$.

\subsection{Bandmerging}

In summary, to bandmerge the available data, we first merged the photometry from all four IRAC channels together with nearIR 2MASS data within each observation (CG4 and Sa101) and then merged together those source lists from each observation. We next included the MIPS data and then the optical data. We now discuss each of these steps in more detail. At the end of this section, we discuss some aggregate statistics of the bandmerged catalog.

To merge the photometry from all four IRAC channels together, we started with a source list from 2MASS. This 
Multiband Measurements of Spitzer-identified YSO Candidates in the CG4+Sa101 Region

\begin{tabular}{|c|c|c|c|c|c|c|c|c|c|c|c|c|c|}
\hline Name & $B(\mathrm{mag})$ & $V(\mathrm{mag})$ & $R_{c}(\mathrm{mag})$ & $I_{c}(\mathrm{mag})$ & $J(\mathrm{mag})$ & $H$ (mag) & $K_{s}(\mathrm{mag})$ & [3.6] (mag) & [4.5] (mag) & [5.8] (mag) & [8.0] (mag) & [24] (mag) & [70] (mag) \\
\hline $073049.1-470209$ & & & & & & & & $18.15 \pm 0.28$ & $16.51 \pm 0.17$ & $14.54 \pm 0.11$ & $12.57 \pm 0.08$ & $8.37 \pm 0.05$ & $>3.40$ \\
\hline $073049.8-465806$ & $\ldots$ & - & $\ldots$ & $\ldots$ & $>17.32$ & $>15.61$ & $15.19 \pm 0.17$ & $14.26 \pm 0.08$ & $13.69 \pm 0.14$ & $12.39 \pm 0.08$ & $11.05 \pm 0.07$ & $7.11 \pm 0.05$ & $>2.87$ \\
\hline $073053.6-465742$ & & & & $\ldots$ & $16.89 \pm 0.18$ & $15.70 \pm 0.10$ & $14.87 \pm 0.11$ & $14.18 \pm 0.08$ & $13.87 \pm 0.09$ & $13.53 \pm 0.09$ & $13.04 \pm 0.09$ & $9.53 \pm 0.09$ & $>5.45$ \\
\hline $073057.5-465611$ & $19.61 \pm 0.06$ & $18.27 \pm 0.06$ & $16.72 \pm 0.06$ & $15.13 \pm 0.06$ & $12.86 \pm 0.02$ & $11.93 \pm 0.02$ & $11.40 \pm 0.02$ & $10.86 \pm 0.07$ & $10.43 \pm 0.07$ & $10.04 \pm 0.07$ & $9.26 \pm 0.07$ & $6.36 \pm 0.05$ & $3.05 \pm 0.22$ \\
\hline $073106.5-465454$ & $x^{2}$ & & 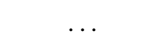 & $\ldots$ & $16.72 \pm 0.14$ & $14.90 \pm 0.05$ & $13.65 \pm 0.04$ & $11.28 \pm 0.07$ & $10.63 \pm 0.07$ & $10.00 \pm 0.07$ & $9.09 \pm 0.07$ & $6.45 \pm 0.04$ & $>2.31$ \\
\hline 073108.4-470130 & $\ldots$ & $\cdots$ & $\ldots$ & $\ldots$ & $15.21 \pm 0.04$ & $14.39 \pm 0.03$ & $13.83 \pm 0.05$ & $13.21 \pm 0.08$ & $12.88 \pm 0.08$ & $12.50 \pm 0.08$ & $11.71 \pm 0.08$ & $8.68 \pm 0.05$ & $>3.21$ \\
\hline $073109.9-465750$ & .. & & & $\ldots$ & $16.55 \pm 0.12$ & $15.71 \pm 0.13$ & $15.09 \pm 0.15$ & $14.35 \pm 0.09$ & $13.91 \pm 0.09$ & $13.52 \pm 0.09$ & $12.82 \pm 0.08$ & $9.70 \pm 0.08$ & $>3.57$ \\
\hline 073110.8-470032 & $17.12 \pm 0.03$ & $15.49 \pm 0.03$ & $14.41 \pm 0.03$ & $13.34 \pm 0.03$ & $11.20 \pm 0.02$ & $10.22 \pm 0.02$ & $9.58 \pm 0.02$ & $8.64 \pm 0.05$ & $8.22 \pm 0.05$ & $7.88 \pm 0.07$ & $7.38 \pm 0.05$ & $4.46 \pm 0.04$ & $2.01 \pm 0.22$ \\
\hline 073114.6-465842 & $\ldots$ & $\ldots$ & $\ldots$ & $\ldots$ & $13.56 \pm 0.03$ & $11.75 \pm 0.03$ & $10.88 \pm 0.02$ & $10.07 \pm 0.08$ & $9.63 \pm 0.07$ & $8.93 \pm 0.07$ & $7.88 \pm 0.07$ & $4.24 \pm 0.04$ & $1.21 \pm 0.22$ \\
\hline 073114.9-470055 & $\ldots$ & & $\ldots$ & $\ldots$ & $15.53 \pm 0.06$ & $14.95 \pm 0.05$ & $14.43 \pm 0.09$ & $13.84 \pm 0.08$ & $13.54 \pm 0.08$ & $13.20 \pm 0.08$ & $12.55 \pm 0.08$ & $9.82 \pm 0.09$ & $>5.41$ \\
\hline 073121.8-465745 & $17.49 \pm 0.05$ & $16.18 \pm 0.05$ & $15.51 \pm 0.05$ & $14.43 \pm 0.05$ & $11.42 \pm 0.03$ & $10.67 \pm 0.2^{\mathrm{a}}$ & $10.24 \pm 0.2^{\mathrm{a}}$ & $9.26 \pm 0.07$ & $8.84 \pm 0.07$ & $8.73 \pm 0.08$ & $8.18 \pm 0.07$ & $5.97 \pm 0.04$ & $>2.53$ \\
\hline 073136.6-470013 & $17.65 \pm 0.04$ & $16.07 \pm 0.04$ & $14.97 \pm 0.04$ & $13.94 \pm 0.04$ & $11.96 \pm 0.02$ & $10.82 \pm 0.03$ & $10.02 \pm 0.02$ & $9.07 \pm 0.08$ & $8.81 \pm 0.05$ & $8.53 \pm 0.05$ & $8.19 \pm 0.05$ & $5.44 \pm 0.04$ & $2.51 \pm 0.22$ \\
\hline 073137.4-470021 & $15.84 \pm 0.04$ & $14.19 \pm 0.04$ & $13.21 \pm 0.04$ & $12.12 \pm 0.04$ & $10.45 \pm 0.02$ & $9.53 \pm 0.02$ & $9.11 \pm 0.02$ & $8.50 \pm 0.08$ & $8.21 \pm 0.05$ & $7.98 \pm 0.05$ & $7.19 \pm 0.05$ & $4.23 \pm 0.04$ & $1.74 \pm 0.22$ \\
\hline $073143.8-465818$ & $19.25 \pm 0.05$ & $18.11 \pm 0.05$ & $16.70 \pm 0.05$ & $15.19 \pm 0.05$ & $13.40 \pm 0.03$ & $12.57 \pm 0.02$ & $12.06 \pm 0.02$ & $11.33 \pm 0.05$ & $10.65 \pm 0.05$ & $9.98 \pm 0.05$ & $8.77 \pm 0.05$ & $5.55 \pm 0.04$ & $>2.53$ \\
\hline 073144.1-470008 & $18.73 \pm 0.05$ & $17.67 \pm 0.05$ & $17.36 \pm 0.05$ & $15.05 \pm 0.05$ & $13.39 \pm 0.05$ & $12.48 \pm 0.07$ & $11.88 \pm 0.03$ & $11.02 \pm 0.05$ & $10.59 \pm 0.05$ & $10.21 \pm 0.05$ & $9.50 \pm 0.05$ & $6.86 \pm 0.04$ & $>1.68$ \\
\hline 073145.6-465917 & $18.95 \pm 0.05$ & $17.28 \pm 0.05$ & $16.04 \pm 0.05$ & $14.60 \pm 0.05$ & $12.96 \pm 0.02$ & $12.04 \pm 0.02$ & $11.71 \pm 0.02$ & $11.44 \pm 0.05$ & $11.27 \pm 0.05$ & $11.02 \pm 0.05$ & $10.21 \pm 0.05$ & $7.47 \pm 0.05$ & $>4.40$ \\
\hline $073326.8-464842$ & $15.24 \pm 0.05$ & $14.11 \pm 0.05$ & $13.35 \pm 0.05$ & $12.64 \pm 0.05$ & $11.49 \pm 0.02$ & $10.74 \pm 0.02$ & $10.35 \pm 0.02$ & $9.91 \pm 0.07$ & $9.70 \pm 0.07$ & $9.43 \pm 0.07$ & $8.56 \pm 0.07$ & $5.14 \pm 0.04$ & $1.83 \pm 0.22$ \\
\hline $073337.0-465455$ & .. & & & $\ldots$ & $15.65 \pm 0.09$ & $15.15 \pm 0.11$ & $14.87 \pm 0.13$ & $14.04 \pm 0.08$ & $13.74 \pm 0.08$ & $13.39 \pm 0.09$ & $12.79 \pm 0.08$ & $10.64 \pm 0.09$ & $>3.60$ \\
\hline 073337.6-464246 & $18.83 \pm 0.02$ & $17.58 \pm 0.02$ & $16.85 \pm 0.02$ & $16.24 \pm 0.02$ & $15.43 \pm 0.07$ & $14.85 \pm 0.10$ & $14.62 \pm 0.11$ & $14.19 \pm 0.08$ & $14.14 \pm 0.09$ & $13.75 \pm 0.09$ & $13.44 \pm 0.11$ & $9.99 \pm 0.12$ & $>6.64$ \\
\hline 073406.9-465805 & $\cdots$ & $\ldots$ & $\ldots$ & $\ldots$ & $14.97 \pm 0.05$ & $14.24 \pm 0.05$ & $13.73 \pm 0.04$ & $13.27 \pm 0.08$ & $12.99 \pm 0.08$ & $12.67 \pm 0.08$ & $11.88 \pm 0.08$ & $8.63 \pm 0.04$ & $>1.68$ \\
\hline $073425.3-465409$ & $\ldots$ & & $\ldots$ & $\ldots$ & $13.44 \pm 0.05^{\mathrm{b}}$ & $12.51 \pm 0.05^{\mathrm{b}}$ & $12.06 \pm 0.06^{\mathrm{b}}$ & $11.73 \pm 0.07$ & $11.44 \pm 0.08$ & $10.94 \pm 0.07$ & $9.82 \pm 0.07$ & $3.54 \pm 0.04$ & \\
\hline 073439.9-465548 & $\ldots$ & $\cdots$ & $\ldots$ & $\ldots$ & $15.98 \pm 0.12$ & $15.03 \pm 0.09$ & $14.19 \pm 0.07$ & $12.99 \pm 0.08$ & $12.33 \pm 0.08$ & $11.72 \pm 0.08$ & $10.90 \pm 0.07$ & $\ldots$ & $\ldots$ \\
\hline
\end{tabular}

Notes.

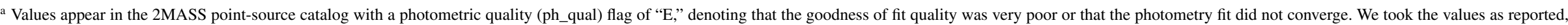
with a large error bar, as additional constraints on the source.

b 2 MASS $J H K_{s}$ photometry comes from the 2MASS extended source catalog, not the point-source catalog; see the discussion in Section 2.4 and Appendix A.21. 
source list includes $J H K_{s}$ photometry and limits, with highquality astrometry. We merged this source list by position to the IRAC-1 source list, using a search radius of $1^{\prime \prime}$, a value empirically determined via experience with other star-forming regions (e.g., Rebull et al. 2010). Objects appearing in the IRAC1 list but not the $J H K_{S}$ list were retained as new potential sources. The master catalog was then merged, in succession, to IRAC-2, 3 , and 4 , again using a matching radius of $1^{\prime \prime}$.

Because we are primarily interested in objects detected by Spitzer, we dropped any objects not having flux densities in at least one Spitzer band (e.g., objects off the edge of the Spitzer maps, having measurements only in 2MASS). Because the source detection algorithm we used can be fooled by instrumental artifacts, we also explicitly dropped objects seen in only one IRAC band as likely artifacts.

For the sources of interest later in the paper, most have counterparts in all three bands in 2MASS by this point in the merging. However, for two sources, this matching failed, at least in part. For one source, 073121.8-465745 (=CG-Ha4), the automatic merging found a counterpart with a measured $J$ magnitude, but the $H K_{s}$ measurements are flagged with a photometric quality (ph_qual) flag of "E," denoting that the goodness of fit quality was very poor, or that the photometry fit did not converge, or that there were insufficient individual data frames for the measurement. Since there was a good measurement at $J$, we assumed that there were sufficient frames at $H K_{s}$, and that something had happened to the fit. We took the values as reported in the catalog, assumed a large error bar, and used these values in the table and plots below; as will be seen in the SED for this object (Figure 15, top center), these values are probably close to what is most appropriate for this object. For source 073425.3465409 , the automatic merging fails, most likely because the 2MASS counterpart is slightly extended, and it may be slightly extended at $3.6 \mu \mathrm{m}$ as well. The nearest match in the catalog is $\sim 2^{\prime \prime}$ away, very large by comparison to other source matches here, but manual inspection strongly suggests that this is the appropriate counterpart to the source seen at Spitzer bands. See Appendix A.21 for more on this interesting source.

We then compared the source lists from the separate observations, CG4 and Sa101, again using a matching radius of $1^{\prime \prime}$. For objects detected in more than one mosaic, we took a weighted average of the flux density at the corresponding band.

Next, we merged the $70 \mu \mathrm{m}$ source list to the $24 \mu \mathrm{m}$ source list. The $70 \mu \mathrm{m}$ PSF is large compared to the positional accuracy needed, and astrophysically, each $70 \mu \mathrm{m}$ source ought to have a counterpart at $24 \mu \mathrm{m}$, given the sensitivity of these observations. We individually verified that each of the $70 \mu \mathrm{m}$ point sources had a $24 \mu \mathrm{m}$ counterpart, and then merged these two source lists. To successfully have the computer match the sources that were clearly matched by eye, a positional accuracy of 2'.5 was required, consistent with our experience in other starforming regions (e.g., Rebull et al. 2010). Since the two MIPS observations do not overlap with each other, no explicit merging of the MIPS source lists from the two observations was required beyond simple concatenation.

To combine the merged MIPS source list into the merged 2MASS+IRAC catalog, we used a positional source match radius of $2^{\prime \prime}$, again determined via experience with other starforming regions (e.g., Rebull et al. 2010). The MOPEX source detection algorithm can be fooled by structure in the nebulosity in the image, and by inspection, this was the case for these data. To weed out these false "sources," we then dropped objects from the catalog that were detections only at $24 \mu \mathrm{m}$ and no other bands. Finally, to merge the $J$ through $70 \mu \mathrm{m}$ catalog to the optical $\left(B V R_{c} I_{c}\right)$ catalog, we looked for nearest neighbors within $1^{\prime \prime}$.

After this entire process, there are $\sim 21,000$ sources with IRAC-1 $(3.6 \mu \mathrm{m})$ or IRAC-2 $(4.5 \mu \mathrm{m})$ detections, $\sim 9000$ sources with IRAC-3 $(5.8 \mu \mathrm{m})$ detections, and $\sim 4000$ sources with IRAC-4 $(8 \mu \mathrm{m})$ detections. About $3000(\sim 15 \%)$ of the IRAC sources have viable data at all four IRAC bands, nearly all of which have counterparts in 2MASS. The optical data do not cover the entire IRAC map, but about half of the four-band IRAC detections have counterparts in the optical catalog. There are only $\sim 500$ sources at $24 \mu \mathrm{m}$ in our catalog and just 11 sources at $70 \mu \mathrm{m}$; note that the MIPS-24 map covers a much smaller area than the IRAC maps, and the MIPS-70 map is smaller still (see Figures 1-7). Ten of the 11 MIPS-70 sources have counterparts at all four IRAC bands; the one that appears to have no four-band IRAC counterpart is saturated in two of the four IRAC bands. About 200 sources have all four IRAC bands plus MIPS-24.

\section{SELECTION OF YSO CANDIDATES WITH INFRARED EXCESS}

With our new multi-wavelength view of the CG4+Sa101 region, we can begin to look for young stars. We focus on finding sources having an infrared excess characteristic of YSOs surrounded by a dusty disk. There is no single Spitzer color selection criterion (or set of criteria) that is $100 \%$ reliable in separating members from non-member contaminants. Many have been considered in the literature (e.g., Allen et al. 2004; Rebull et al. 2007; Harvey et al. 2007; Gutermuth et al. 2008, 2009; Rebull et al. 2010, 2011). Some make use of just MIPS bands, some make use of just IRAC bands, most use a series of color criteria, and where possible, they make use of (sometimes substantial) ancillary data. In our case of the CG4+Sa101 region, we have some ancillary data, but the bulk of the data are IRAC+2MASS data. In this case, the best choice for selecting YSO candidates is the approach developed by Gutermuth et al. $(2008,2009)$ and adapted by Guieu et al. $(2009,2010)$ for the case in which no extinction map is available. This selection method starts from the set of objects detected at all four IRAC bands and uses 2MASS and MIPS data where possible. It implements a series of color cuts to attempt to remove contaminants such as background galaxies and knots of nebulosity.

When we impose these IRAC-based color cuts, we find 25 potential YSO candidates. We then inspected each of these in all available images and color-color and color-magnitude diagrams (CMDs). On the basis of this inspection, we dropped three of the 25 potential YSO candidates off of our list, though additional data will be needed to be sure that these objects are extragalactic. Two of those dropped objects (073542.2-470126 and 073548.5-470727) have no available data other than IRAC, their SEDs are very flat, and they are located near the edges of our images, far from other YSOs and nebulosity. We suspect that these are extragalactic contaminants. The third object, 073243.5-464941, is returned by the IRAC selection as having a small excess at 5.8 and $8 \mu \mathrm{m}$. It is seen at 2MASS and IRAC bands, but is undetected at $24 \mu \mathrm{m}$ to a fairly stringent limit (971 $\mu \mathrm{Jy}$ or $9.67 \mathrm{mag}$ ). If it has an excess at $24 \mu \mathrm{m}$, it is a very small excess. The $70 \mu \mathrm{m}$ data do not cover this object, so there are no constraints (not even limits) at $70 \mu \mathrm{m}$. Moreover, it is a relatively faint source next to a very bright source, located far 


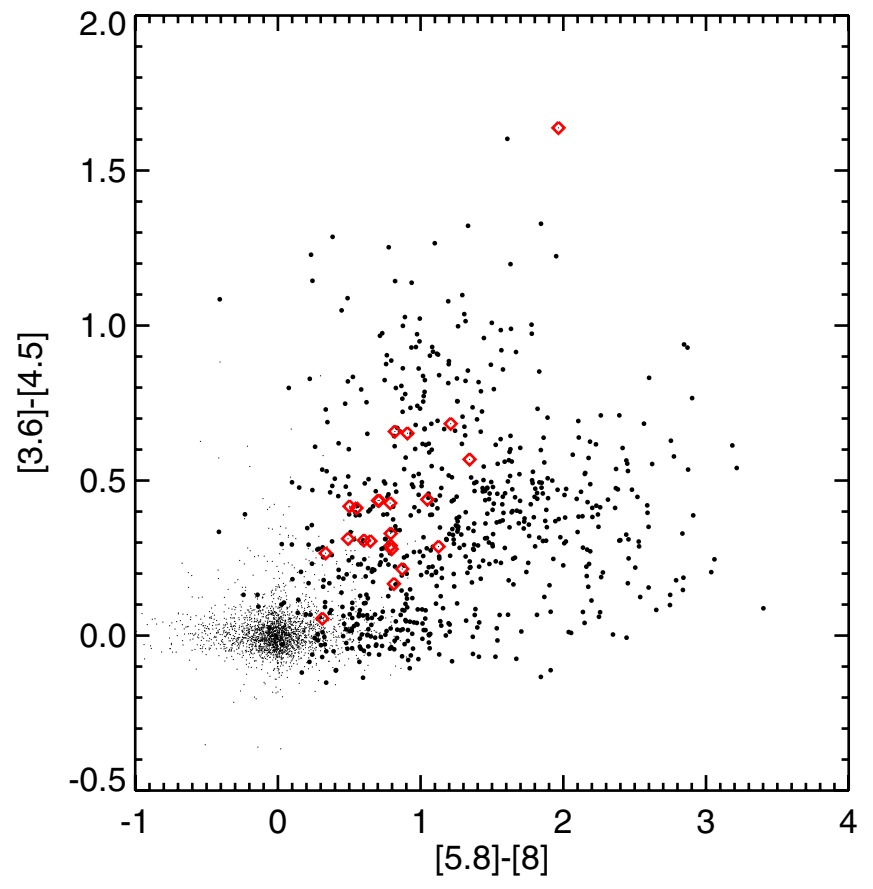

Figure 8. [3.6]-[4.5] vs. [5.8]-[8] color-color diagram for CG4+Sa101. Small dots are objects in the catalog; larger dots are objects identified as contaminants, and large red diamonds highlight our YSO candidates. All of our Spitzer-selected YSO candidates have colors in this diagram consistent with known YSOs, but many contaminants do too.

(A color version of this figure is available in the online journal.)

from any nebulosity. The wings of the bright source are likely to adversely affect the photometric accuracy of the measurements associated with this object. Because of this uncertainty and the very low excess as measured, we have dropped this object from our YSO candidate list as a likely foreground or background star.

The remaining 22 YSO candidates that pass the color cuts are shown in Figure 8. In this figure, objects with zero color are likely foreground or background stars (photospheres without disks), though some could be young stars that have already shed their disks. Young stars with circumstellar disks are generally red in both IRAC colors, but contaminants such as galaxies also may have these colors. All of the 22 objects highlighted in this figure have IRAC colors consistent with young stars with disks.

The Gutermuth et al. (2008, 2009) selection criteria have provisions for adding stars to the list of candidate YSOs based on properties at other bands, such as MIPS bands. We now investigate the properties of objects in our catalogs at the MIPS bands to see if we should add additional objects to our list of YSO candidates. In summary of the rest of this section, while we find some interesting objects, in the end, we do not add any more YSO candidates to our list.

Young stars having inner disk holes and thus excesses at only the longest bands can be revealed via comparison of the $24 \mu \mathrm{m}$ measurement to a shorter band, such as $K_{\mathrm{s}}$ or [3.6]. If the data are available, one should use [3.6] versus [3.6]-[24] rather than $K_{s}$ versus $K_{s}-$ [24]. There is an intrinsic spread in $K_{s}-$ [24] photospheric colors that is not present in [3.6]-[24] because late-type stars are not colorless at $K_{s}-$ [24] (Gautier et al. 2007). The effects of reddening are stronger at $K_{\mathrm{s}}$ than at $3.6 \mu \mathrm{m}$, and if $2 \mathrm{MASS}$ is the only source of $K_{\mathrm{s}}$, even short $3.6 \mu \mathrm{m}$ integrations can reach fainter sources than 2MASS does. In our case of CG4+Sa101, the IRAC coverage is larger than the

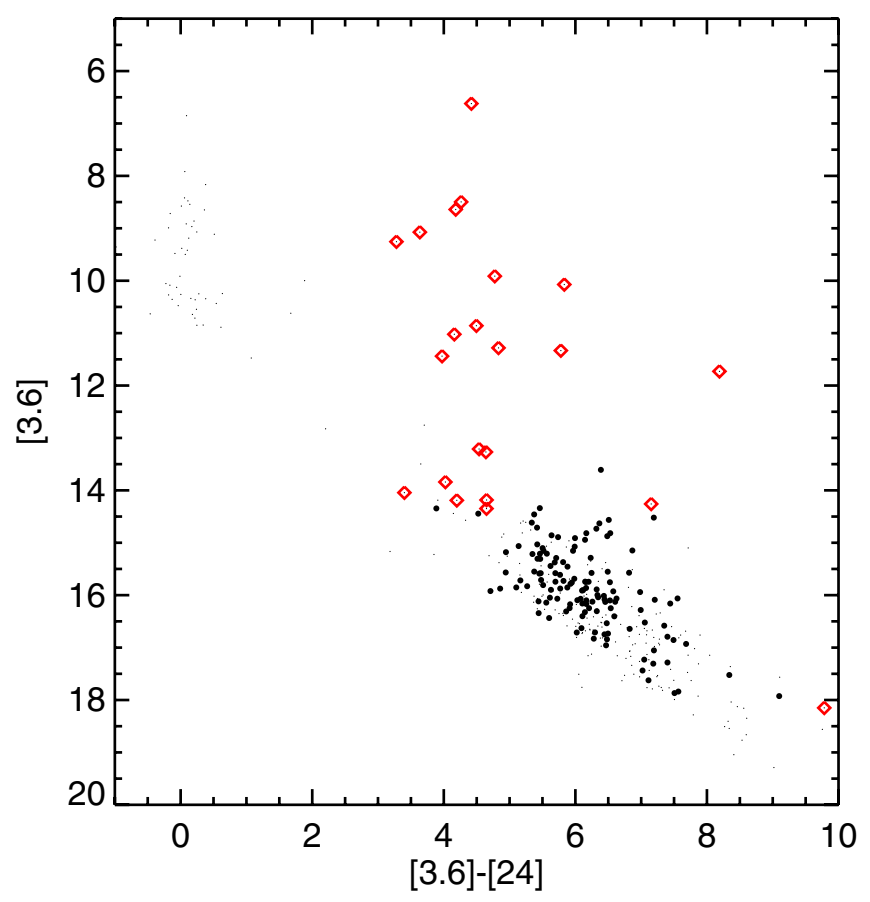

Figure 9. [3.6] vs. [3.6]-[24] CMD for CG4+Sa101. Small dots are objects in the catalog; larger dots are objects identified as contaminants, and large red diamonds highlight our IRAC-selected YSO candidates. All of the IRACselected YSO candidates have colors in this diagram consistent with known YSOs.

(A color version of this figure is available in the online journal.)

MIPS coverage, and so we use [3.6] versus [3.6]-[24] to look for any objects with an excess starting at $24 \mu \mathrm{m}$.

Figure 9 shows this [3.6] versus [3.6]-[24] diagram, with the same notation as in the prior figure. Ordinary stellar photospheres (likely foreground or background stars) have [3.6] $-[24] \sim 0$, and galaxies make up the large, elongated source concentration near $[3.6]-[24] \sim 6,[3.6] \sim 16$. Objects not in this region, e.g., the brighter and/or redder objects, are less likely to be part of the Galactic or extragalactic backgrounds, and more likely to be YSOs with a $24 \mu \mathrm{m}$ excess. Most of the IRAC-identified YSO candidates are indeed in the region of this diagram occupied by other known YSOs (see, e.g., Rebull et al. 2010, 2011; Guieu et al. 2009, 2010). There is one (073049.1-470209) that is among the reddest objects in this diagram, near [3.6]-[24] 10; see Appendix A.1 for more on this specific object. Most of the objects already ruled out as YSO candidates based on their IRAC properties are in the extragalactic concentration of sources. The objects with [3.6]-[24] $\sim 0$ do not have apparent excesses, but there are eight additional objects with [3.6]-[24] $>1$ and [3.6] $<14.5$ that seem to have the right placement in this diagram to be YSO candidates. We investigated each of these candidates, and none had evidence based on SED shape, significance of excess, or appearance in the images compelling enough to have us add them to our list of YSO candidates. The apparent small excesses just at $24 \mu \mathrm{m}$ are most likely due to source confusion at the lower resolution $24 \mu \mathrm{m}$ band, with either a background source or a low-mass companion. The most compelling one based on the SED is $073355.0-464838$, but the source seems to be confused with a nearby source that emerges at $8 \mu \mathrm{m}$, and we strongly suspect that the $24 \mu \mathrm{m}$ flux instead corresponds to the object appearing at $8 \mu \mathrm{m}$, rather than the point source seen at $8 \mu \mathrm{m}$ and shorter bands. We do not add this source to our list of 


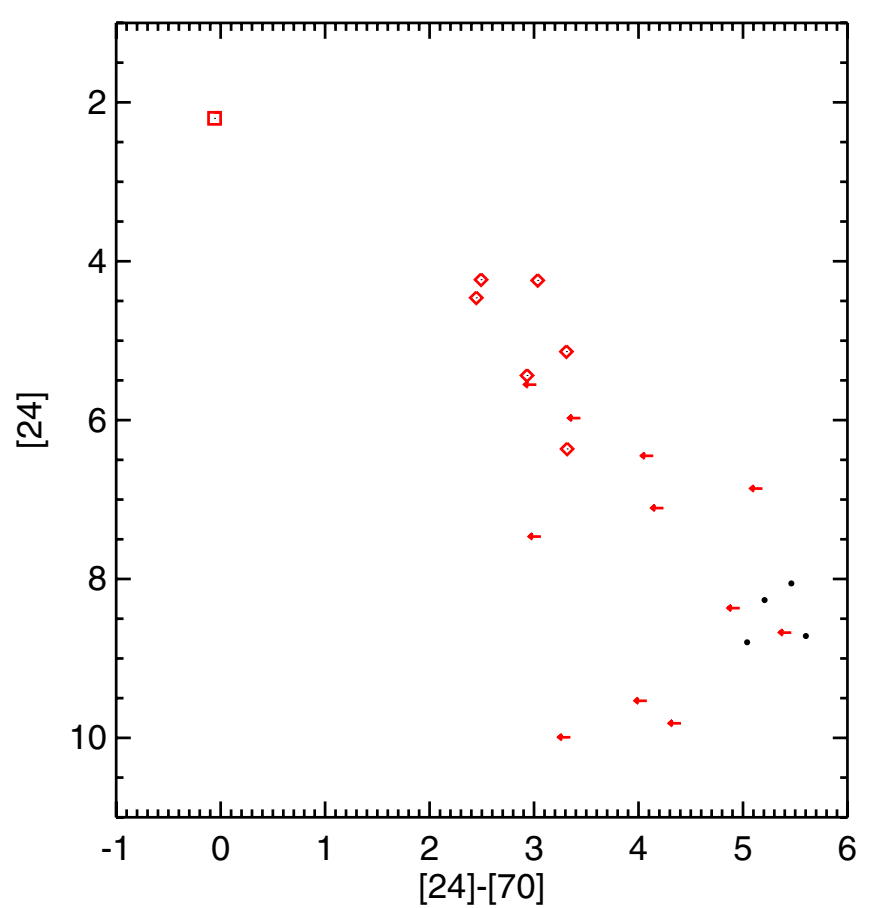

Figure 10. [24] vs. [24]-[70] CMD for CG4+Sa101. As in prior plots, dots are objects identified as contaminants, and large red diamonds highlight our IRAC-selected YSO candidates. A red square highlights a very bright likely background star; see the text. The arrows indicate the positions of the YSO candidates for which we could obtain upper limits at $70 \mu \mathrm{m}$. The IRAC-selected YSO candidates have colors in this diagram consistent with known YSOs.

(A color version of this figure is available in the online journal.)

YSO candidates at this time. Higher spatial resolution $24 \mu \mathrm{m}$ observations would be required to resolve this issue.

Some of the brightest stars in the $\mathrm{CG} 4+\mathrm{Sa} 101$ region are saturated in at least the first two IRAC bands, so neither of the YSO search mechanisms we have used thus far would find them. However, these sources could be YSOs, and they are not all saturated in MIPS bands. As our last attempt to search for YSO candidates with infrared excesses, Figure 10 shows the [24] versus [24]-[70] diagram for our region. This diagram for this region is sparse, but a better-populated diagram (see, e.g., Rebull et al. 2010) would basically resemble Figure 9, with photospheres being bright and colorless (having [24]-[70] 0), and galaxies being red and faint. In Figure 10, the few points that are available include things that are galaxies (things that have been ruled out as contaminants based on IRAC) which are in the extragalactic part of the diagram here as well, six detections that have colors consistent with YSOs, many limits for our YSO candidates, and one thing (square in Figure 10) that is too bright to be a likely galaxy, and does not appear to be particularly red in this diagram. This very bright object is 073339.7-464839, and its SED suggests at first glance that it might be a YSO with a small excess just at 24 and $70 \mu \mathrm{m}$. It is detected at $J H K_{s}$, [5.8], and [8], and the $K_{s}$, [5.8], and [8] measurements are all consistent with one Rayleigh-Jeans tail, and the [24] and [70] measurements are offset on a different, redder Rayleigh-Jeans tail, as might be consistent with a small thermally heated dust disk. However, it is a very bright object and saturated at the shortest two IRAC bands; the photometry at $J H K_{s}$ [5.8][8] may also be compromised beyond what our formal errors suggest. It is matched in Simbad to IRAS 07321-4642. We suspect that this is a background asymptotic giant branch star, or another sort of

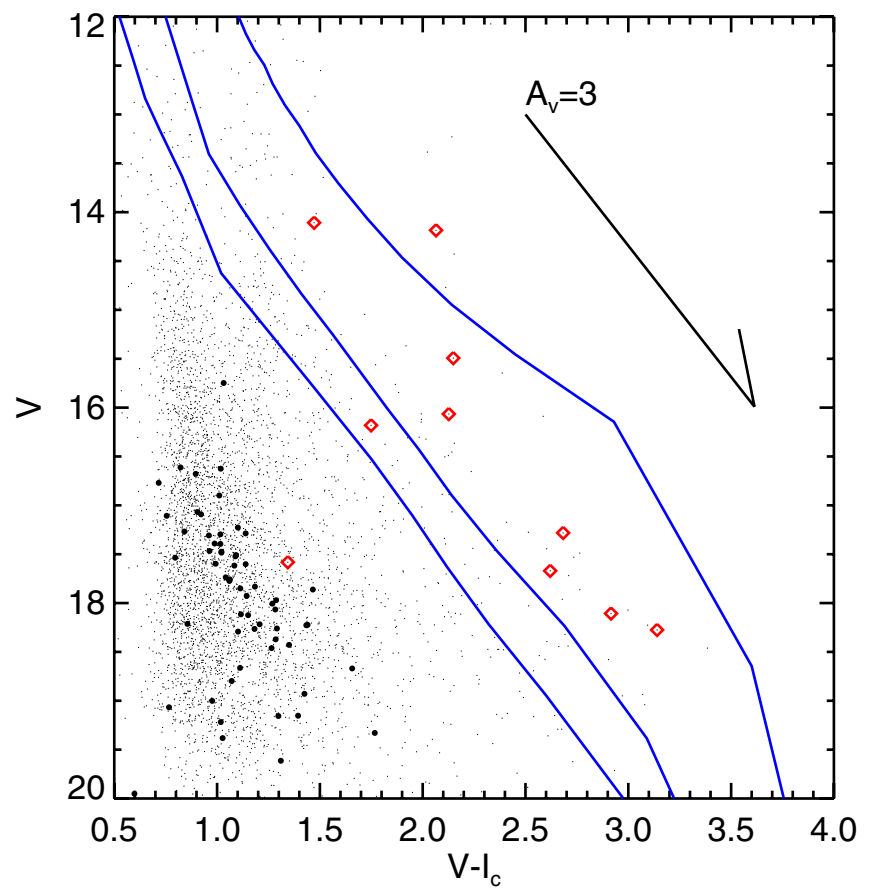

Figure 11. $V$ vs. $\left(V-I_{c}\right) \mathrm{CMD}$ for $\mathrm{CG} 4+\mathrm{Sa} 101$. Small dots are objects in the catalog, larger dots are objects identified as contaminants, and large red diamonds highlight our YSO candidates. Isochrones given are models from Siess et al. (2000) at 1, 10, and $30 \mathrm{Myr}$, scaled to $500 \mathrm{pc}$, where we have tuned the color-effective temperature relation such that the $100 \mathrm{Myr}$ isochrone matches that of the Pleiades single-star sequence (Stauffer et al. 2007; Jeffries et al. 2007). A reddening vector is also indicated. All of the YSO candidates shown here, except for one, are in the region occupied by young stars at 300-500 pc.

(A color version of this figure is available in the online journal.)

bright background giant, and not a legitimate YSO candidate. We do not include it on our YSO candidate list.

We move ahead from here with the 22 IRAC-selected YSO candidates and now investigate their multiband properties.

\section{PROPERTIES OF SELECTED YSO CANDIDATES}

\subsection{Optical Properties}

Optical data can greatly aid in confirming or refuting YSO candidacy because they provide constraints on the Wien side of the SED. In Guieu et al. (2010), most of the IRAC-selected candidates in IC 2118 proved to be too faint, most vividly in the optical, to be likely cluster members. Just 10 of our candidate YSOs have optical data available, and they appear in Figure 11. The objects with optical data that have already been ruled out as YSOs based on their IRAC properties are all well below the Siess et al. (2000) 30 Myr isochrone scaled to $500 \mathrm{pc}$. One YSO candidate object appears below the $30 \mathrm{Myr}$ isochrone; 073337.6-464246 is within the distribution of clear non-YSO points. We do not remove this object from our list, since a variety of reasons (such as scattered light) could result in a YSO appearing below the $30 \mathrm{Myr}$ isochrone; for more discussion of this object, please see Appendix A.19. As noted above, the distance to this association is uncertain; if the isochrones are instead scaled to $300 \mathrm{pc}$, then one other object (073121.8-465745, a previously identified YSO) appears to be just below the $30 \mathrm{Myr}$ isochrone instead of just above it.

Deeper optical data are desirable in order to obtain magnitude estimates for the remaining YSO candidates. 


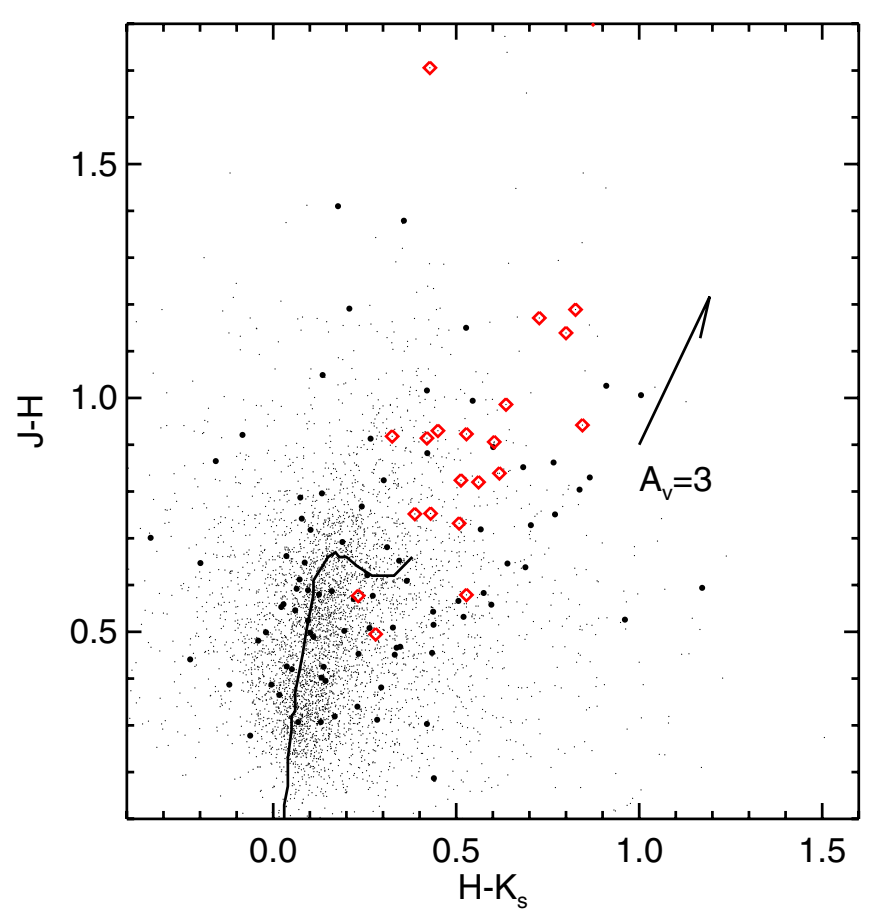

Figure 12. $J-H$ vs. $H-K_{s}$ diagram for the sample, with the same notation as earlier figures. The main sequence is indicated by a solid line. Most of the YSO candidates have an infrared excess starting at the $H$ band with moderate reddening.

(A color version of this figure is available in the online journal.)

\subsection{Near-IR Properties}

Near-IR data can also aid in confirming or refuting YSO candidacy. Since we do not have spectral types for most of our sources, it is difficult to estimate the degree of reddening. Figure 12 shows $J-H$ versus $H-K_{s}$ for the sample. This plot suggests that most of our YSO candidates have an infrared excess with a moderate degree of reddening.

\subsection{B-band Properties}

Young stars that are actively accreting from their circumstellar disk can have excess ultraviolet emission in the $U$ or $B$ bands, or even longer bands during periods of intense accretion. However, these bands are also most sensitive to reddening. Figure 13 shows $B-V$ versus $R_{c}-I_{c}$ for the sample, with the main sequence indicated as a solid line. Stars with a $B$-band excess and relatively small values of $A_{V}$ would be blue, e.g., below the line in this figure. Objects above the line on the upper right of this figure are pushed into that location by high $A_{V}$. At least four of the YSO candidates have a $B$ excess, most likely from mass accretion; four more appear to have been pushed from the region of clear $B$ excess by high $A_{V}$. Similar results for the same objects are obtained from the $B-V$ versus $V-I_{c}$ plot. The individual objects are listed in the Appendix.

\subsection{Spectral Energy Distributions}

Coordinates and our measured magnitudes between $B$ and $70 \mu \mathrm{m}$ for our $22 \mathrm{YSO}$ candidates appear in Table 2. Six of them $(27 \%)$ are rediscoveries of the previously known YSOs in this region from Table 1. Figures 14-16 are the SEDs for the YSO candidates.

To guide the eye, we wished to add reddened stellar models to the SEDs, but spectral types are known only for

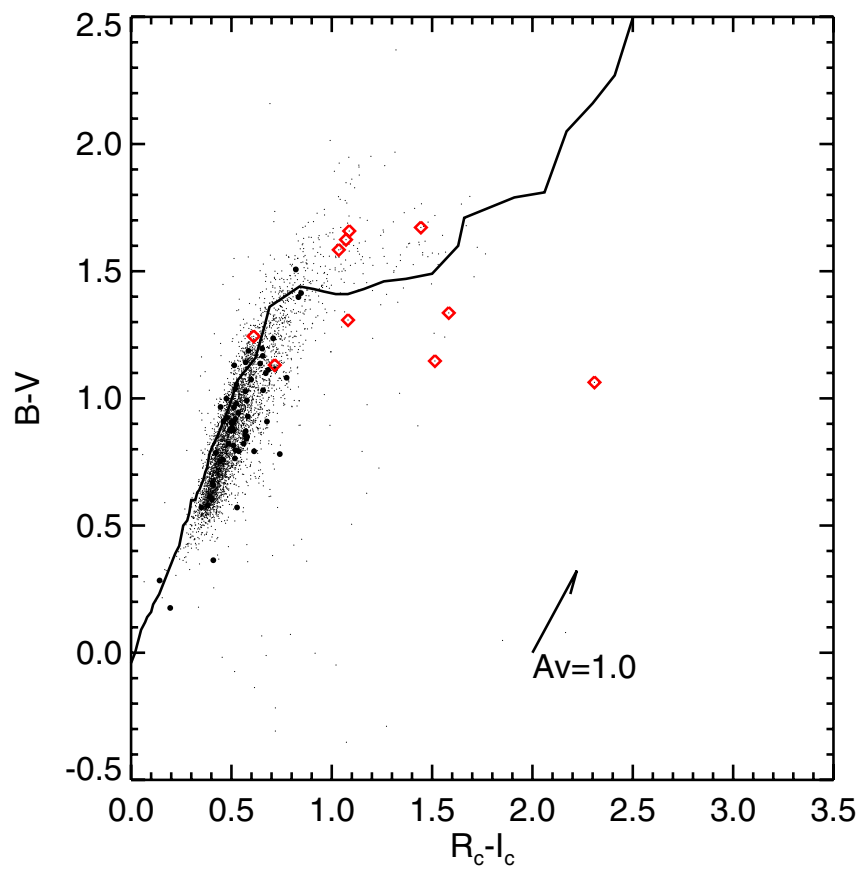

Figure 13. $B-V$ vs. $R_{c}-I_{c}$ for the sample, with the same notation as earlier figures. The main sequence is indicated by a solid line. Stars with a $B$-band excess and relatively small values of $A_{V}$ would be blue, e.g., below the line in this figure. Objects above the line on the upper right of this figure are pushed into that location by high $A_{V}$. At least four and probably eight of the YSO candidates have a $B$ excess, most likely from mass accretion.

(A color version of this figure is available in the online journal.)

the six previously known YSOs. In order to provide a reference for the remaining objects, we assumed a spectral type of M0. For each object, a reddened model is shown, selected from the Kurucz-Lejeune model grid (Lejeune et al. 1997, 1998) and normalized to the $K_{s}$ band where possible (and to the closest band otherwise). Note that this is not meant to be a robust fit to the object, but rather a representative stellar SED to guide the eye such that the infrared excesses are immediately apparent. In some cases, ultraviolet excesses may also be present. Additional spectroscopic observations are needed to better constrain these fits.

In the spirit of Wilking et al. (2001), we define the near- to mid-IR slope of the SED, $\alpha=d \log \lambda F_{\lambda} / d \log \lambda$, where $\alpha>0.3$ for a Class I, 0.3 to -0.3 for a flat-spectrum source, -0.3 to -1.6 for a Class II, and $<-1.6$ for a Class III. For each of the YSO candidate objects in our sample, we performed a simple ordinary least-squares linear fit to all available photometry (just detections, not including upper or lower limits) between 2 and $24 \mu \mathrm{m}$, inclusive. Note that errors on the infrared points are so small as to not affect the fitted SED slope. The precise definition of $\alpha$ can vary, resulting in different classifications for certain objects. Classification via this method is provided specifically to enable comparison within this paper via internally consistent means. Note that the formal classification puts no lower limit on the colors of Class III objects (thereby including those with SEDs resembling bare stellar photospheres, and allowing for other criteria to define youth). By searching for IR excesses, we are incomplete in our sample of Class III objects. The classes for the YSO (previously known and candidate) sample appear in Table 3. Out of the 22 stars, $16(73 \%)$ are Class II.

Based on the SEDs and location in several color-color and color-magnitude diagrams, we have ranked the YSO candidates 

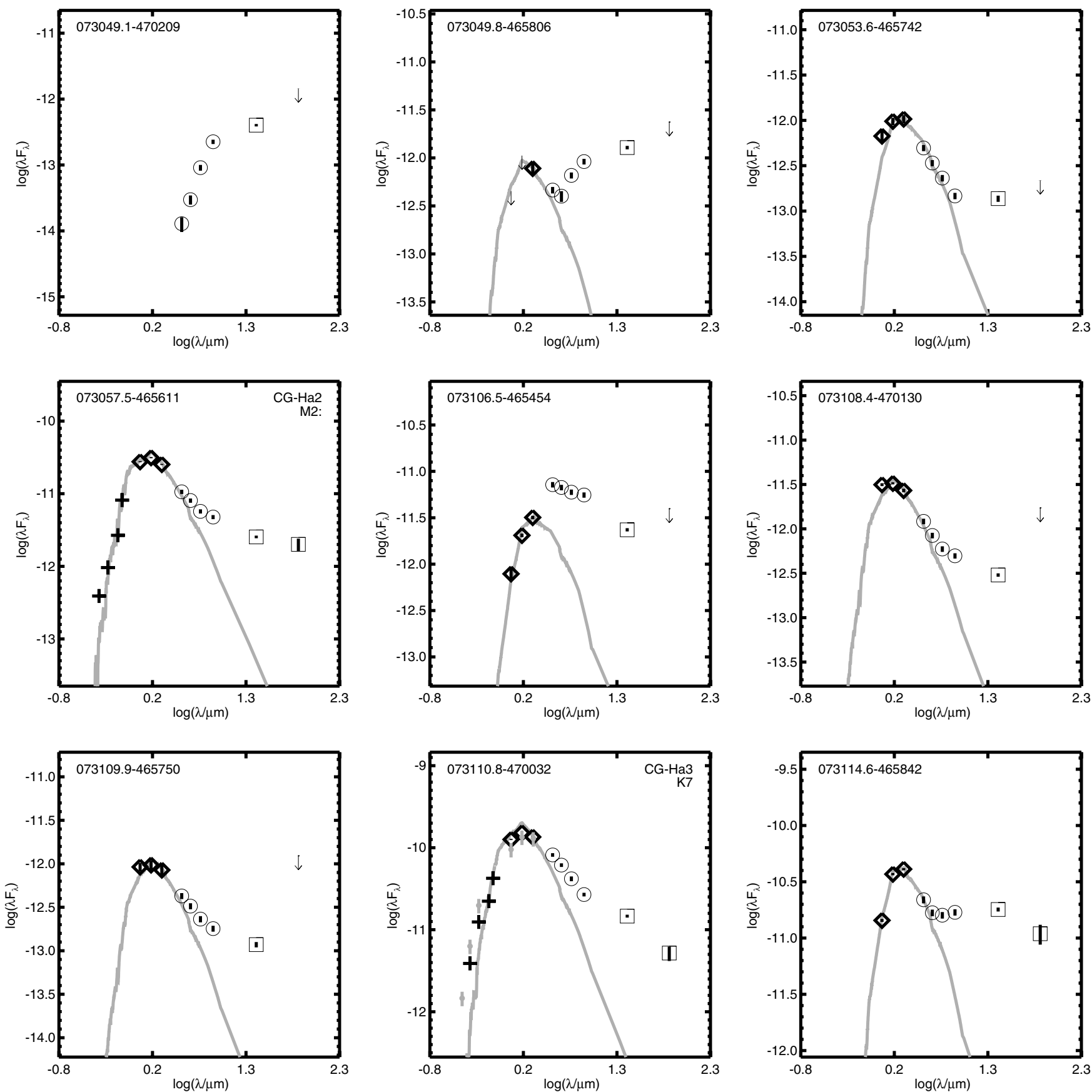

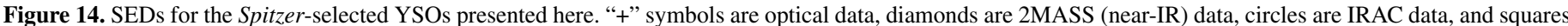

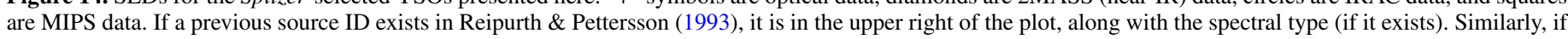

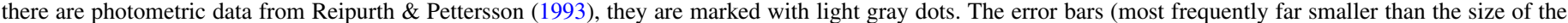

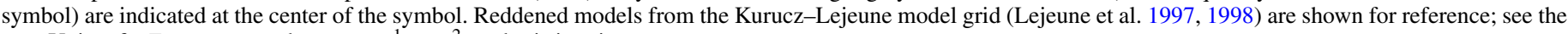
text. Units of $\lambda F_{\lambda}$ as presented are erg s $\mathrm{s}^{-1} \mathrm{~cm}^{-2}$, and $\lambda$ is in microns.

loosely into three bins: high likelihood of being YSOs (grade A), mid-grade quality (grade B), and relatively low likelihood of being YSOs (grade $\mathrm{C}$ ). This grade also appears in Table 3. Most of them (16) are in the grade A bin, which includes the six previously identified ones. Comments on individual objects (including justifications for the grades that were given) appear in the Appendix.

Figure 17 shows the $8 \mu \mathrm{m}$ mosaic with the positions of the YSO candidates overlaid, color coded by YSO quality. Most of the grade A and B objects are clustered near the previously known YSOs. The Sa101 region has a relatively tight clumping of most (16) of the YSOs, with a median nearest neighbor distance of $\sim 62^{\prime \prime}$. The CG4 region has six YSOs, much less tightly clumped, with a median nearest neighbor distance nearly five times larger, $\sim 301^{\prime \prime}$. Clustering is also very commonly found among young stars, so especially in the case of the Sa101 association, the fact that they are clustered also bolsters the case that they are legitimate YSOs.

Because the distance to this association is uncertain, we looked at whether the relative placement of stars in the optical CMD could be used to constrain the distance to the stars. Figure 18 presents the $M_{V}$ versus $V-I \mathrm{CMD}$, comparing 

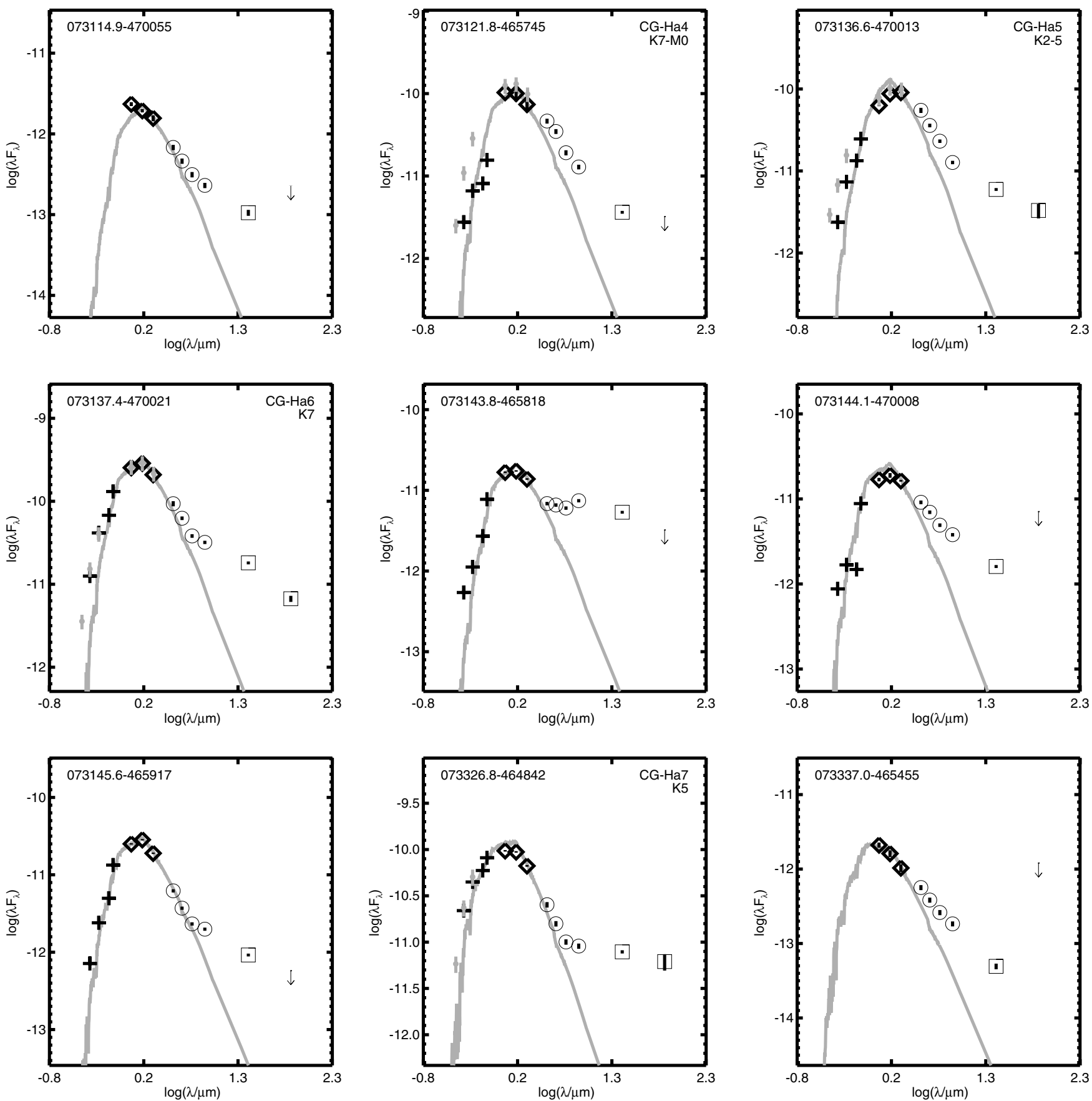

Figure 15. SEDs, continued. Notation as in the previous figure.

CG4+Sa101 stars to YSOs in Taurus (with data from Rebull et al. 2010; Güdel et al. 2007 and references therein). Based on morphological grounds (e.g., the degree to which the YSOs are still embedded in their natal gas), we expect that the CG4+Sa101 stars might be slightly younger than the often more physically dispersed Taurus stars. On the other hand, based on the ratio of Class I to Class II sources, the CG4+Sa101 objects might be slightly older than Taurus. In Figure 18, there are not many CG4+Sa101 objects, and the distribution is broad, but assuming a distance of $500 \mathrm{pc}$, then CG4+Sa101 appears to be quite comparable in age to Taurus at $\sim 3$ Myr. Assuming a distance of $300 \mathrm{pc}$, the CG4+Sa101 stars are on the whole older than the Taurus stars. Figure 18 thus weakly suggests that CG4+Sa101 is farther rather than closer.

\section{THE GALAXY}

The galaxy in our field, ESO 257-G 019, has not been the target of any case studies before. The galaxy is classified as type "SB(s)cd?" in the NASA Extragalactic Database (NED). We measure a major axis length of 3.3 or $34.7 \mathrm{kpc}$ at the assumed distance of $36.1 \mathrm{Mpc}$ at the $3 \sigma$ level above the noise in our channel 1 image. We also measured a minor axis length of 0.8 or $8.4 \mathrm{kpc}$ in the same image for this highly inclined galaxy. The surface brightness distribution is close to exponential within the central $3 \mathrm{kpc}$. The galaxy has a clumpy structure at larger distances from its center, with the most prominent clumps appearing about $26^{\prime \prime}$ and $73^{\prime \prime}$ (along the major axis) to the northwest and $26^{\prime \prime}$ to the southeast (just outside of the 

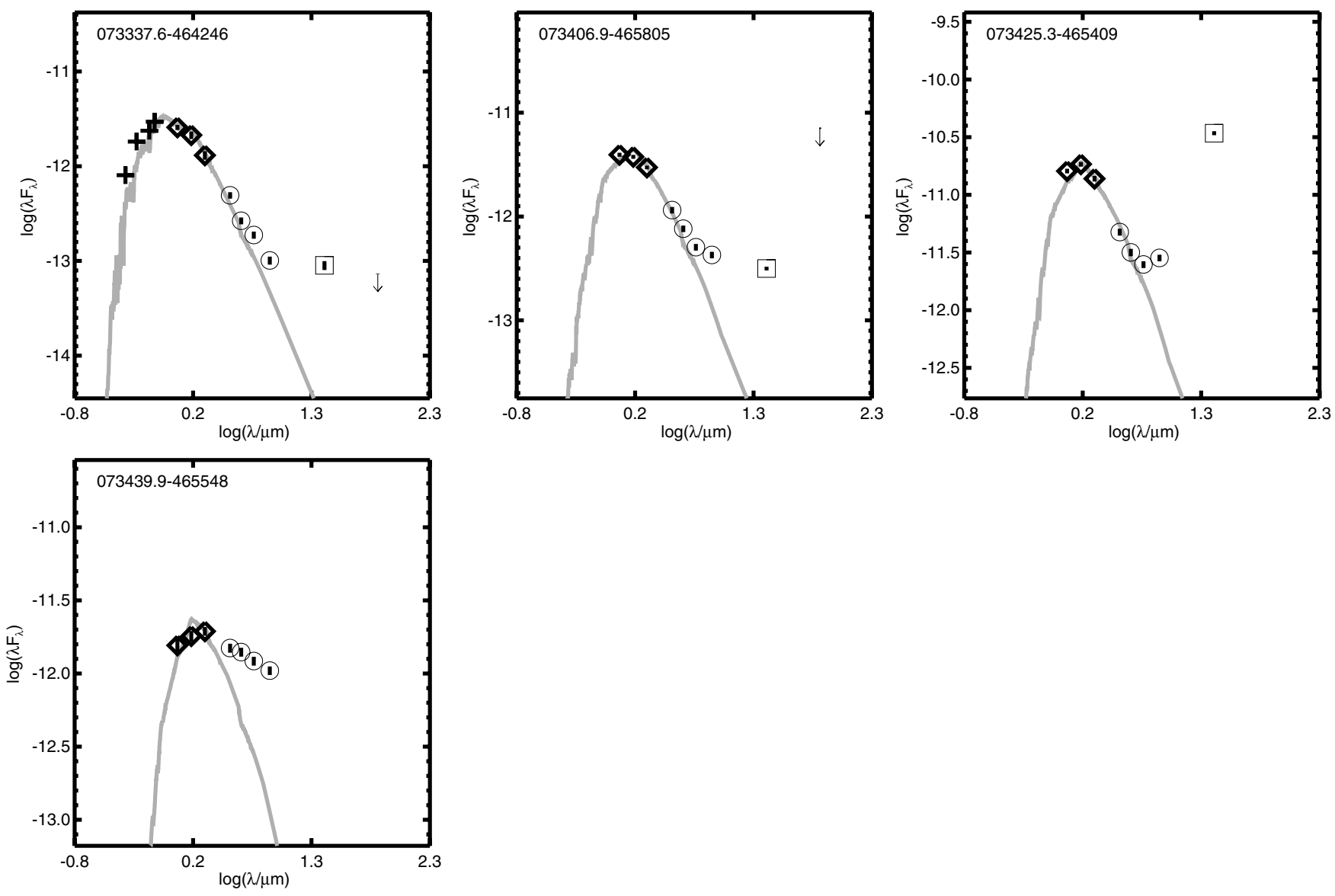

Figure 16. SEDs, continued. Notation as in the previous figure.

Table 3

Final List of YSO Candidates in the CG4+Sa101 Region

\begin{tabular}{lllllll}
\hline \hline Name & Syn. & Sp.Ty. & Class & Quality & Region & \\
\hline $073049.1-470209$ & & & I & C & Sa101 & Reddest and faintest in [3.6] vs. [3.6]-[24] \\
$073049.8-465806$ & & I & B & Sa101 & \\
$073053.6-465742$ & & II & A & Sa101 & Small excess at $8 \mu$ m; most of the excess at 24 $\mu$ m \\
$073057.5-465611$ & CG-Ha2 & M2: & II & A+ & Sa101 & Apparently lowest mass object in this list \\
$073106.5-465454$ & & & flat & C & Sa101 & Somewhat discontinuous SED \\
$073108.4-470130$ & & II & A & Sa101 & \\
$073109.9-465750$ & & & II & A & Sa101 & \\
$073110.8-470032$ & CG-Ha3 & K7 & II & A+ & Sa101 & High $A_{V}$ likely \\
$073114.6-465842$ & & flat & A & Sa101 & \\
$073114.9-470055$ & & II & A & Sa101 & \\
$073121.8-465745$ & CG-Ha4 & K7-M0 & II & A+ & Sa101 & \\
$073136.6-470013$ & CG-Ha5 & K2-5 & II & A+ & Sa101 & \\
$073137.4-470021$ & CG-Ha6 & K7 & II & A+ & Sa101 & \\
$073143.8-465818$ & & & flat & A & Sa101 & \\
$073144.1-470008$ & & II & A & Sa101 & \\
$073145.6-465917$ & & II & A & Sa101 & Inner disk hole? \\
$073326.8-464842$ & CG-Ha7 & K5 & II & A+ & CG4 & \\
$073337.0-465455$ & & II & B & CG4 & Very low in optical CMD; near edges of maps \\
$073337.6-464246$ & & II & C & CG4 \\
$073406.9-465805$ & & II & A & CG4 & \\
$073425.3-465409$ & & I & A & CG4 & Extended in optical, NIR \\
$073439.9-465548$ & & & II & CG4 & Sparse SED \\
\hline
\end{tabular}

Notes. ${ }^{\text {a }}$ This grade is meant to indicate rough confidence in the likelihood that the given YSO candidate is a legitimate YSO. Previously identified YSOs are given a grade of "A+," our highest-quality YSO candidates are grade "A," our mid-grade YSO candidates are grade "B," and our lowest-confidence YSO candidates are grade "C." For discussion of individual objects (and an explanation of why each object has that grade), please see the Appendix. 


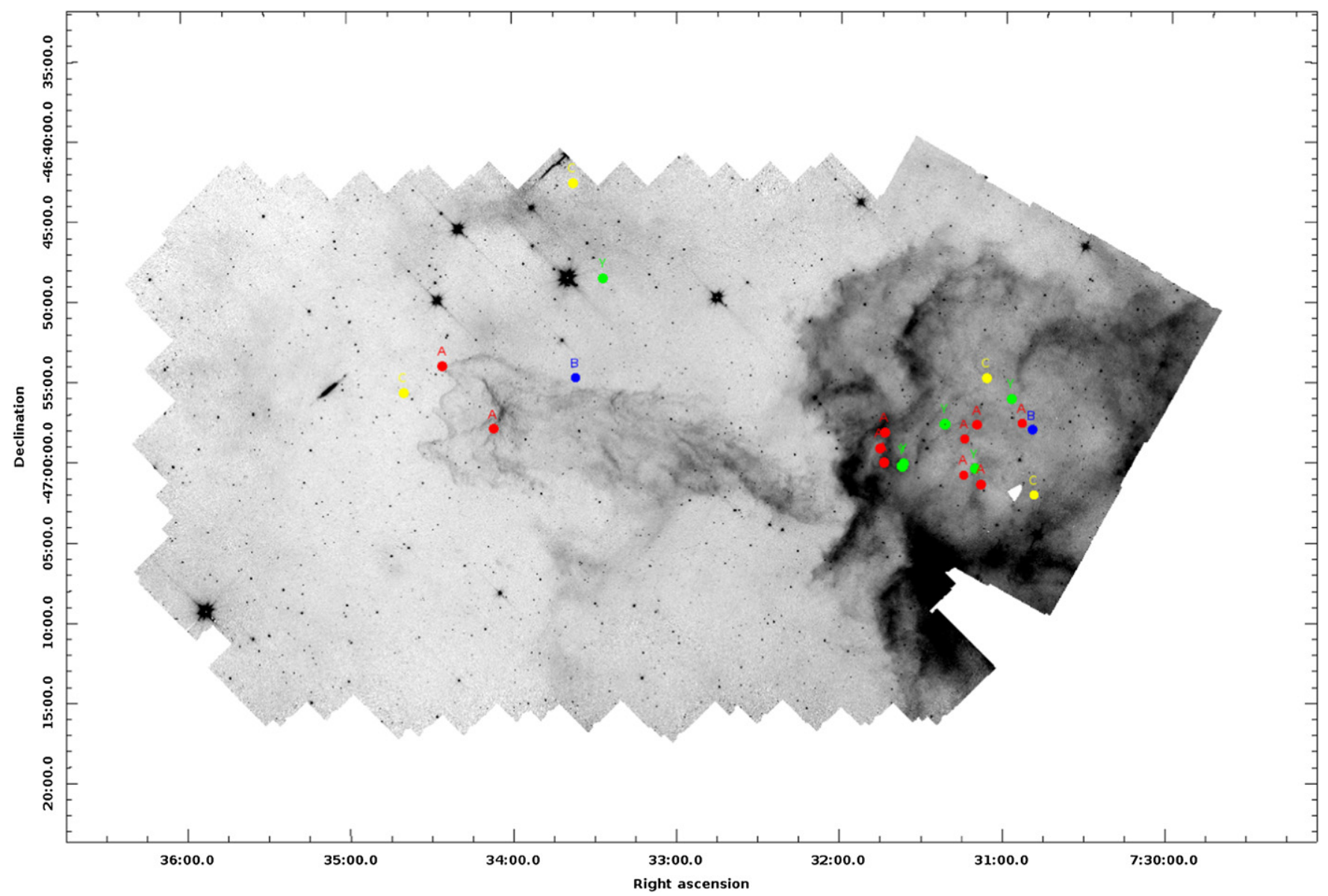

Figure 17. Reverse gray-scale mosaic of $8 \mu \mathrm{m}$ data with the locations of the YSOs (previously known and new candidates) indicated, color coded by YSO quality grade. Previously known YSOs are indicated by a green dot and a "Y," high-quality (grade "A") YSOs are indicated by a red dot and an "A," mid-quality (grade "B") YSOs are indicated by a blue dot and a "B," and low-quality (grade "C") YSOs are indicated by a yellow dot and a "C."

plane of the galaxy). We also measured the IRAC [3.6]-[4.5] and [3.6]-[8.0] colors by using all the pixels above the $10 \sigma$ level. The [3.6]-[4.5] color is 0.0 and the [3.6]-[8.0] color is 1.9 , both close to the typical values for late-type galaxies, as determined by Pahre et al. (2004), and consistent with the galaxy classification as given in NED. ESO 257-G 019 appears to be a fairly isolated galaxy, with no nearby companions within our mapped areas.

\section{CONCLUSIONS}

We used Spitzer Space Telescope data from the Spitzer Heritage Archive to search for new candidate young stars in the CG4+Sa101 region of the Gum Nebula. This region appears to be actively forming young stars, perhaps as a result of the passage of an ionization front from the stars powering the Gum Nebula (Reipurth \& Pettersson 1993). We rediscovered all six of the previously identified young stars in our maps as having excesses at Spitzer bands. We have also discovered 16 entirely new young star candidates based on their Spitzer properties. We used optical ground-based data and near-infrared data from 2MASS to help constrain the SEDs of these new young star candidates. We have sorted the new young star candidates into grades of confidence that they are, in fact, legitimate new young stars. We find 16 high confidence (grade "A") objects, including the six previously identified YSOs, two mid-grade confidence (grade "B"), and four low-grade confidence (grade "C") young star candidates. For all of the new young star candidates, though, additional data will be needed, such as optical photometry where it is missing, and optical spectroscopy to obtain spectral types (and rule out extragalactic contamination). Most of the new objects are clustered in the Sa101 region, and most are SED Class II.

This work was performed as part of the NASA/IPAC Teacher Archive Research Program (NITARP; http://nitarp.ipac.caltech. edu), class of 2010. We acknowledge here all of the students and other folks who contributed their time and energy to this work and the related poster papers presented at the 2011 January American Astronomical Society (AAS) meeting in Seattle, WA. They include-with V. Hoette: C. Gartner, J. VanDerMolen, L. Gamble, L. Matsche, A. McCartney, M. Doering, S. Brown, R. Wernis, J. Wirth, and M. Berthoud; with C. Johnson: R. Crump, N. Killingstad, T. McCanna, S. Caruso, A. Laorr, K., Mork, E. Steinbergs, and E. Wigley; with C. Mallory: N. Mahmud.

We thank J. R. Stauffer for helpful comments on the manuscript. J. S. Kim and M. Foster thank the NASA Space Grant program at the University of Arizona.

This research has made use of NASA's Astrophysics Data System (ADS) Abstract Service, and of the SIMBAD database, operated at CDS, Strasbourg, France. This research has made use of data products from the Two Micron All Sky Survey (2MASS), which is a joint project of the University of Massachusetts and the Infrared Processing and Analysis Center, 


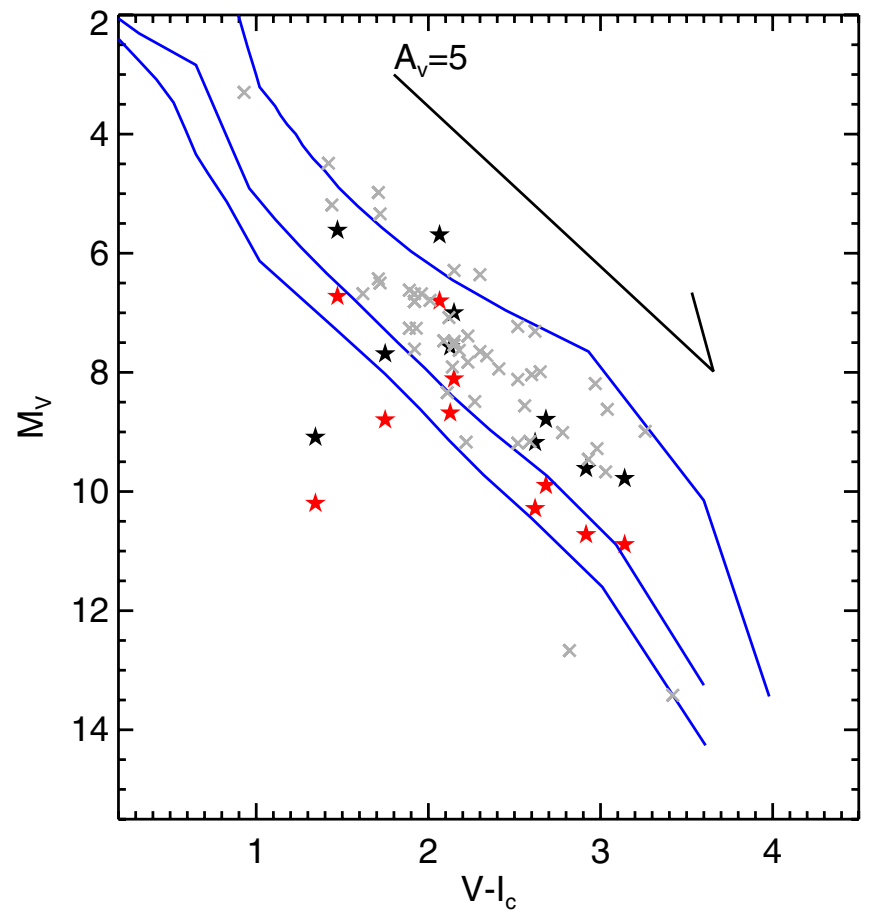

Figure 18. Optical $M_{V}$ vs. $V-I_{C}$ CMD. The Siess et al. (2000) isochrones are included $(1,10$, and $30 \mathrm{Myr})$, but shifted to absolute $M_{V}$. The black stars are our YSO candidates, assuming a distance of $500 \mathrm{pc}$, and the red stars are our YSO candidates, assuming a distance of 300 pc. The gray $\times$ symbols are Taurus YSOs (from Rebull et al. 2010; Güdel et al. 2007, and references therein), taken to be at $140 \mathrm{pc}$ (Torres et al. 2007, 2009). The Taurus distribution is broad and there are many fewer CG4+Sa101 stars, but this distribution weakly suggests that $\mathrm{CG} 4+\mathrm{Sa} 101$ is farther rather than closer (see the text).

funded by the National Aeronautics and Space Administration and the National Science Foundation. These data were served by the NASA/IPAC Infrared Science Archive, which is operated by the Jet Propulsion Laboratory, California Institute of Technology, under contract with the National Aeronautics and Space Administration. This research has made use of the Digitized Sky Surveys, which were produced at the Space Telescope Science Institute under U.S. Government grant NAG W-2166. The images of these surveys are based on photographic data obtained using the Oschin Schmidt Telescope on Palomar Mountain and the UK Schmidt Telescope. The plates were processed into the present compressed digital form with the permission of these institutions. This research has made use of the NASA/ IPAC Extragalactic Database (NED) which is operated by the Jet Propulsion Laboratory, California Institute of Technology, under contract with the National Aeronautics and Space Administration.

The research described in this paper was partially carried out at the Jet Propulsion Laboratory, California Institute of Technology, under contract with the National Aeronautics and Space Administration.

\section{APPENDIX}

\section{COMMENTS ON INDIVIDUAL OBJECTS}

\section{A.1. 073049.1-470209}

This object, 073049.1-470209, the westernmost in the set, is also the reddest source in the set. It is the YSO candidate farthest in the upper right of the IRAC color-color diagram (Figure 8) and is the reddest and faintest YSO in [3.6] versus [3.6]-[24]
(Figure 9). Based on its SED (Figure 14), it is not surprising that there are no optical or even 2MASS counterparts; it very steeply falls from $24 \mu \mathrm{m}$ back to $3.6 \mu \mathrm{m}$, and it seems that very deep integrations would be needed to obtain measurements of this object at shorter wavelength bands. It is not detected at $70 \mu \mathrm{m}$, but it is in a high background region, so the $70 \mu \mathrm{m}$ limit is not very constraining. This type of SED can be found in very young, very embedded, very low mass objects, but also in extragalactic contaminants. Because of its proximity to the rest of the Sa101 objects, it remains in the list, but we have given it the lowestquality grade $(C)$. Its steep SED means that it is one of the three Class I objects in our list. Additional follow-up data are needed to determine the true nature of this source.

\section{A.2. $073049.8-465806$}

This object, 073049.8-465806, is relatively faint at $3.6 \mu \mathrm{m}$, at least in comparison to the rest of the YSO candidates here. It appears on the top edge of the clump of likely extragalactic sources in the [3.6] versus [3.6]-[24] plot (Figure 9). It is only detected at $K_{s}$ in 2MASS with limits at $J$ and $H$. Based on the SED, it looks quite extinguished, e.g., there seems to be high $A_{V}$ in the direction of this source. It is located in close (projected) proximity to other YSOs and YSO candidates; YSOs are also frequently found near other YSOs. It is not detected at $70 \mu \mathrm{m}$, but the limit is shallow enough that it does not provide a strong constraint on the SED. It has an SED characteristic of YSOs, and that plus the apparently high $A_{V}$ plus its location in the cloud close to other YSOs has yielded a "B" grade. The steep rise of the SED from 4.5 to $24 \mu \mathrm{m}$ influences the classification slope fitting such that it is one of the three Class I objects in our list. Additional follow-up data are needed.

\section{A.3. $073053.6-465742$}

Object 073053.6-465742 has 2MASS data, but no available optical data, and just a limit at $70 \mu \mathrm{m}$. The SED (Figure 14) suggests that there is high $A_{V}$ toward this source. Given our overly simple modeling, there seems to be a small but significant excess at $8 \mu \mathrm{m}$ (better modeling is required to confirm this), and there is an apparently large excess at $24 \mu \mathrm{m}$. Whenever an apparent excess is seen only at $24 \mu \mathrm{m}$, because the MIPS-24 camera has lower spatial resolution than the shorter bands, there is a risk that the flux density measured at $24 \mu \mathrm{m}$ is contaminated by source confusion, most likely with a nearby background source, but it could also be with a low-mass companion to the young star candidate. There is only a limit at $70 \mu \mathrm{m}$ to help constrain the SED, though it is located in close (projected) proximity to other YSOs and YSO candidates. In this case, the fact that the excess appears to be significant at at least two bands, its proximity to other nearby Sa101 sources, and the likelihood that there is high $A_{V}$ toward this source suggests that it may be a legitimate YSO, so we have placed it in the highest-quality source bin (grade "A"), and it is a Class II. Spectroscopy of this target is required to determine whether or not it is a YSO.

$$
\text { A.4. } 073057.5-465611=C G-H a 2
$$

CG-H $\alpha 2$ was identified in Reipurth \& Pettersson (1993) as a YSO. It has counterparts at all bands we considered here, including $70 \mu \mathrm{m}$. In the optical (Figure 11), it is in the locus of young stars above the $30 \mathrm{Myr}$ isochrone, and it appears there to be the lowest mass object of the YSO candidates with optical data. The spectral type given in Reipurth \& Pettersson (1993) is M2:, so that is the model we have used in Figure 14. It is also 
one of the stars with a clear $B$ excess in Figure 13, which can also be noted in the SED itself. Such an excess is a characteristic of active accretion. Because it is a previously identified YSO, it appears as grade "A+" in Table 3; it is a Class II.

\section{A.5. $073106.5-465454$}

073106.5-465454 is the northernmost YSO candidate within the Sa101 association. It has a 2MASS counterpart, but the SED (Figure 14) seems somewhat "disjointed" between the 2MASS and IRAC portions. This could be from very high extinction, or intrinsic stellar variability between the epochs of observation at the NIR and MIR, or source confusion. It is detected at $24 \mu \mathrm{m}$ but there is only a limit at $70 \mu \mathrm{m}$. We categorize it as a "C"-grade YSO candidate, with a "flat" SED class. Additional data are needed to confirm or refute the YSO status of this object.

\section{A.6. $073108.4-470130$}

This object, 073108.4-470130, has an SED (Figure 14) quite consistent with other known YSOs, although there are no optical data and just a limit at $70 \mu \mathrm{m}$. If it is really a young star, it has a significant excess in at least 8 and $24 \mu \mathrm{m}$, and probably $5.8 \mu \mathrm{m}$ as well. We categorize it as a very high quality YSO candidate (“A”), SED Class II.

\section{A.7. $073109.9-465750$}

Like the prior object, 073109.9-465750 has an SED (Figure 14) quite consistent with other known YSOs, again without optical data. There is a limit at $70 \mu \mathrm{m}$, and it is in a high background region, so there is little constraint on the SED. Like the prior object (073108.4-470130), if it is really a young star, it has a significant excess in at least two bands. We categorize it as a very high quality YSO candidate ("A"), SED Class II.

$$
\text { A.8. } 073110.8-470032=C G-H a 3
$$

CG-H $\alpha 3$ is another YSO identified in Reipurth \& Pettersson (1993) and has some photometry reported there as well. It is detected in all bands considered here, $B$ through $70 \mu \mathrm{m}$. In the optical (Figure 11), it is well within the clump of YSO candidates above the $30 \mathrm{Myr}$ isochrone. As seen in its SED (Figure 14), the optical and near-IR data from Reipurth \& Pettersson (1993) are quite consistent with the optical and near-IR data we report here, with some weak evidence for variability in the optical. Reipurth \& Pettersson (1993) report a type of K7, and that is the model we have used in Figure 14. Like CG-H $\alpha 2$, the SED suggests that there may be some $B$-band excess (most likely due to accretion); in Figure 13, it appears as one of the objects apparently pushed above the main sequence due to reddening. Because it is a previously identified YSO, it appears as grade "A+" in Table 3; it is a Class II.

\section{A.9. $073114.6-465842$}

This object can be seen at all four IRAC bands and both of the MIPS bands considered here. It also has a 2MASS counterpart, but no optical data. The SED (Figure 14) suggests that there is relatively high $A_{V}$ toward this object. Of the new YSO candidates that appear to have some photometric points on the photosphere, this object appears to have the highest $A_{V}$. If this object is actually a young star, it has an IR excess in four bands. We categorize this as another highest-quality (grade "A") YSO candidate, with SED class "flat." Additional data are needed, including optical photometry.

\section{A.10.073114.9-470055}

Another new YSO candidate, 073114.9-470055, has a 2MASS counterpart but no optical data and just a limit at $70 \mu \mathrm{m}$ (Figure 15). If it is a legitimate young star, it has an IR excess in more than two bands. We categorize it as a grade "A" YSO candidate, SED Class II. Additional data are needed, including optical photometry.

\section{A.11. 073121.8-465745 =CG-Ha4}

CG-H $\alpha 4$ is another YSO from Reipurth \& Pettersson (1993) and has some photometry reported there as well as a spectral type of K7-M0. As discussed above, this object has a highquality $J$ measurement in the 2 MASS point-source catalog, but the $H K_{s}$ measurements were flagged as having low-quality photometry. We accepted the low-quality measurements with a large uncertainty and added them to the SED in Figure 15. Given the normalization as seen there, there could be a disk excess beginning as early as $3.6 \mu \mathrm{m}$, but with a better measurement at the near-IR and thus a better constraint on the location of the photosphere, the disk excess could start at a longer wavelength, and we suspect that it probably does. The optical data we report, as compared with the optical data from Reipurth \& Pettersson (1993), suggest substantial intrinsic source variability, a common characteristic of young stars. In the optical CMD in Figure 11, it is apparently the oldest YSO of the set of YSO candidates above the 30 Myr isochrone. This object's apparent intrinsic variability could move it around in this diagram; moreover, the uncertain distance to the CG4+Sa101 cloud moves this object above or below the $30 \mathrm{Myr}$ isochrone (as discussed above). This is the only previously known YSO in our set that is not detected at $70 \mu \mathrm{m}$. It appears in Figure 13 as having one of the smallest $B$-band excesses, but the photometry as seen in Figure 15 suggests that perhaps its placement in Figure 13 could be improved. Because it is a previously identified YSO, it appears as grade "A+" in Table 3; it is a Class II.

\section{A.12.073136.6-470013=CG-Ha5}

CG-H $\alpha 5$ is reported as a K2-K5 in Reipurth \& Pettersson (1993). It is detected at all available bands we discuss here. It appears in Figure 13 as having a $B$-band excess and subject to high $A_{V}$. Because it is a previously identified YSO, it appears as grade "A+" in Table 3; it is a Class II.

\section{A.13. 073137.4-470021 = CG-Ha6}

$\mathrm{CG}-\mathrm{H} \alpha 6$, located very close to $\mathrm{CG}-\mathrm{H} \alpha 5$, is reported as a $\mathrm{K} 7$ in Reipurth \& Pettersson (1993). It is detected at all available bands we discuss here, $B$ through $70 \mu \mathrm{m}$. The infrared excess appears to start around $8 \mu \mathrm{m}$, suggesting a possible inner disk hole; more detailed modeling is needed to be sure. It appears in Figure 13 as having a $B$-band excess and subject to high $A_{V}$. It is another "A+," Class II, in Table 3.

\section{A.14. 073143.8-465818}

This object, 073143.8-465818, is detected at optical through $24 \mu \mathrm{m}$. It is a high-quality YSO candidate (grade "A"), and SED class "flat." In the optical (Figure 11), it is the lowest mass YSO candidate without a prior identification. It has a clear $B$ excess in Figure 13. It is not detected at $70 \mu \mathrm{m}$. Spectroscopy of this object is required to confirm/refute its YSO status and obtain an initial guess at its mass. 


\section{A.15. 073144.1-470008}

073144.1-470008 is another high-quality YSO candidate (grade "A," Class II), detected in the optical through $24 \mu \mathrm{m}$. It too has a clear $B$ excess in Figure 13. It is undetected at $70 \mu \mathrm{m}$.

\section{A.16. 073145.6-465917}

073145.6-465917 is the last of the YSO candidates in this list associated with Sa101. It is a high-quality (grade "A") YSO candidate, detected at optical through $24 \mu \mathrm{m}$, but with only a limit at $70 \mu \mathrm{m}$. Based on the approximate SED fit in Figure 15, the disk excess starts at $8 \mu \mathrm{m}$, though additional modeling (and a spectral type) is required to be sure. In Figure 13, it appears as having a $B$-band excess and subject to $A_{V}$. It has an SED Class of II.

\section{A.17. 073326.8-464842=CG-Ha7}

$\mathrm{CG}-\mathrm{H} \alpha 7$ is the only previously known YSO in the CG4 region covered by our maps. Reipurth \& Pettersson (1993) report a K5 spectral type; their optical data are quite consistent with the optical data we report. We detect this object at all available bands reported here, $B$ through $70 \mu \mathrm{m}$. In Figure 11, it is the highest apparent mass YSO candidate, though reddening can strongly influence this placement. The disk excess (Figure 15) begins at the longer IRAC bands, suggesting a possible inner disk hole. It does not appear to have a $B$-band excess in Figure 13. It is located far from any nebulosity (Figure 17), as noted by Reipurth \& Pettersson (1993). It is an SED Class II.

\section{A.18. 073337.0-465455}

Object 073337.0-465455 has counterparts at $J$ through $24 \mu \mathrm{m}$; there are no optical data available. Its placement in the [3.6] versus [3.6]-[24] diagram (Figure 9) suggests that it is at the edge of our detection limits; it appears at [3.6]-[24] 3.4, [3.6] 14. It is undetected at $70 \mu \mathrm{m}$. If it is a legitimate YSO, it has an excess in at least three bands. It is located just off the northern edge of the globule. We place it in the "grade B" bin, and it is SED Class II. Additional follow-up data are needed.

\section{A.19. 073337.6-464246}

This object, 073337.6-464246, is detected at optical through $24 \mu \mathrm{m}$, but not at $70 \mu \mathrm{m}$. It is the candidate seen in Figure 11 as very low in the optical CMD, and closest to the IRAC photospheric locus of points in Figure 8. There is a marginal excess seen at 5.8 and $8 \mu \mathrm{m}$, and then a larger excess at $24 \mu \mathrm{m}$; as discussed above, excesses seen only (or primarily) in a single point at $24 \mu \mathrm{m}$ can be a result of source confusion with adjacent sources. This source is also located very far from any nebulosity, near the edges of some of our IRAC maps (Figure 17). We place this marginal candidate as a grade "C"; it is an SED Class II. Additional data are needed.

\section{A.20.073406.9-465805}

073406.9-465805 is the only YSO candidate of our set located projected onto the globule/elephant trunk. It appears to also be projected onto a bright rim at $8 \mu \mathrm{m}$ (Figure 17), so it is potentially being revealed now by the action of the ionization front. There are no optical or $70 \mu \mathrm{m}$ counterparts, but there are counterparts at $J$ through $24 \mu \mathrm{m}$. If it is a young star, there is an IR excess at more than two bands. We place it as a grade "A," Class II object, and additional data are needed.

\section{A.21. 073425.3-465409}

073425.3-465409 is a very interesting source. In the [3.6] versus [3.6]-[24] diagram (Figure 9), it is the brightest, reddest object ([3.6] 11.7, [3.6]-[24] 8.2), and it is located right on the "lip" of the globule (Figure 17), in a region where YSOs might be expected to form. None of the other "fingers" of the molecular cloud have apparent associations with infrared objects. After the automatic bandmerging described in Section 2 above, this object did not appear to have a $2 \mathrm{MASS}$ counterpart. However, in looking at the overall shape and brightness of the SED, we suspected that it should have a match in 2MASS. Examining the images by eye, there is clearly a source at this location visible in 2MASS and the Palomar Observatory Sky Survey (POSS) images. At the POSS bands, it is distinctly fuzzy. It is also slightly resolved at $J$; it has been identified with 2MASX J07342550-4654106. The position given for this source in the 2MASS point-source catalog is 2 .' 1 away from the position in the IRAC catalog, and the position from the 2MASS extended source catalog is $1^{\prime \prime} .98$ away from the IRAC position, both of which are very large compared to most of the rest of the catalog. If it is also slightly resolved in IRAC, this could affect the positional uncertainty. Certainly, the structure of the molecular cloud around this source is complex and could also have affected the position in the catalog. In any case, manual inspection of the images ensures that the object is really the same in the two catalogs, so the $J H K_{s}$ photometry from the extended source catalog was attached to this Spitzer source. The SED (Figure 16) rises steeply at the long wavelengths, but it is unfortunately just off the edge of the $70 \mu \mathrm{m}$ maps.

Given this object's SED and location, we have classified this as a high-quality (grade "A") YSO candidate, with SED Class I. Additional data are required to confirm or refute this object's status.

\section{A.22.073439.9-465548}

073439.9-465548, the last object in our list, has counterparts at $J$ through only $8 \mu \mathrm{m}$, and as such, it has a sparse SED. (It is off the edge of both the 24 and $70 \mu \mathrm{m}$ maps.) It is located just to the east of the end of the globule, consistent with it having been relatively recently uncovered. The shape of the IRAC portion of the SED is a little different than that for other objects in this set; despite the negative overall slope of the IRAC points, none of the IRAC points seem to be photospheric (compare to other SEDs in Figures 14-16), and there is even a very slight negative curvature (the slope between 3.6 and $4.5 \mu \mathrm{m}$ is slightly shallower than that between 4.5 and $5.8 \mu \mathrm{m}$ ). On the basis of experience looking at many hundreds of SEDs for YSOs and contaminants (Rebull et al. 2010, 2011), we have some reservations about the shape of this SED; without additional photometric data, it is hard to give this object a high grade. We give this one a " $\mathrm{C}$ " grade; it is a Class II.

\section{REFERENCES}

Allen, L. E., et al. 2004, ApJS, 154, 363

Bok, B. J., \& Reilly, E. F. 1947, ApJ, 105, 255

Fazio, G., et al. 2004, ApJS, 154, 10

Franco, G. A. P. 1990, A\&A, 227, 499

Gautier, T. N., et al. 2007, ApJ, 667, 527

Güdel, M., et al. 2007, A\&A, 468, 353

Guieu, S., et al. 2009, ApJ, 697, 787

Guieu, S., et al. 2010, ApJ, 720, 46 
Gutermuth, R., Megeath, S. T., Myers, P. C., Allen, L. E., Pipher, J. L., \& Fazio, G. G. 2009, ApJS, 184, 18

Gutermuth, R., et al. 2008, ApJ, 674, 336

Haikala, L. K., Mäkelä, M. M., \& Väisänen, P. 2010, A\&A, 522, 106

Harvey, P., et al. 2007, ApJ, 663, 1139

Hawarden, T. G., \& Brand, P. W. J. L. 1976, MNRAS, 175, 19

Jeffries, R., Oliveira, J., Naylor, T., Mayne, N., \& Littlefair, S. 2007, MNRAS, 376,580

Kim, J. S., Walter, F., \& Wolk, S. 2003, in The Future of Cool-Star Astrophysics: 12th Cambridge Workshop on Cool Stars, Stellar Systems, and the Sun, ed. A. Brown, G. M. Harper, \& T. R. Ayres (Boulder, CO: Univ. Colorado), 799

Laher, R., Gorjian, V., Rebull, L., Masci, F., Fowler, J., Helou, G., Kulkarni, S., \& Law, N. 2011a, PASP, submitted

Laher, R., et al. 2011b, PASP, submitted

Landolt, A. 1992, AJ, 104, 340

Lejeune, T., Cuisinier, F., \& Buser, R. 1997, A\&AS, 125, 229

Lejeune, T., Cuisinier, F., \& Buser, R. 1998, A\&AS, 130, 65

Makovoz, D., \& Marleau, F. 2005, PASP, 117, 1113

Mink, D. 1997, in ASP Conf. Ser. 125, Astronomical Data Analysis Software and Systems VI, ed. G. Hunt \& H. E. Payne (San Francisco, CA: ASP), 249

Osterbrock, D. 1957, ApJ, 125, 622

Pahre, M. A., Ashby, M. L. N., Fazio, G. G., \& Willner, S. P. 2004, ApJS, 154, 235
Pettersson, B. 2008, in Handbook of Star Forming Regions, Volume II: The Southern Sky ASP Monograph Publications, Vol. 5, ed. B. Reipurth (San Francisco, CA: ASP), 43

Pozzo, M., Jeffries, R., Naylor, R., Totten, E., Harmer, S., \& Kenyon, M. 2000, MNRAS, 313, L23

Rebull, L., et al. 2007, ApJS, 171, 447

Rebull, L., et al. 2010, ApJS, 186, 259

Rebull, L., et al. 2011, ApJS, 193, 25

Reipurth, B. 1983, A\&A, 117, 183

Reipurth, B., \& Pettersson, B. 1993, A\&A, 267, 439

Rieke, G., et al. 2004, ApJS, 154, 25

Sandqvist, A. 1976, MNRAS, 177, 69

Sandqvist, A. 1977, A\&A, 57, 467

Siess, L., Dufour, W., \& Forestini, M. 2000, A\&A, 358, 593

Skrutskie, M., et al. 2006, AJ, 131, 1163

Stauffer, J., et al. 2007, ApJS, 172, 663

Torres, R. M., Loinard, L., Mioduszewski, A. J., \& Rodríguez, L. F. 2007, ApJ, 671,1813

Torres, R. M., Loinard, L., Mioduszewski, A. J., \& Rodríguez, L. F. 2009, ApJ, 698,242

Werner, M., et al. 2004, ApJS, 154, 1

Wilking, B., Bontemps, S., Schuler, R., Greene, T., \& Andre, P. 2001, ApJ, 551, 357 